\title{
Synthesis, Structure, Theoretical Studies and Ligand Exchange Reactions of Monomeric, T-shaped Arylpalladium(II) Halide Complexes with an Additional, Weak Agostic Interaction
}

James P. Stambuli and John F. Hartwig*

Department of Chemistry, Yale University, P.O. Box 208107, New Haven, Connecticut 06520-8107

\author{
Supporting Information
}




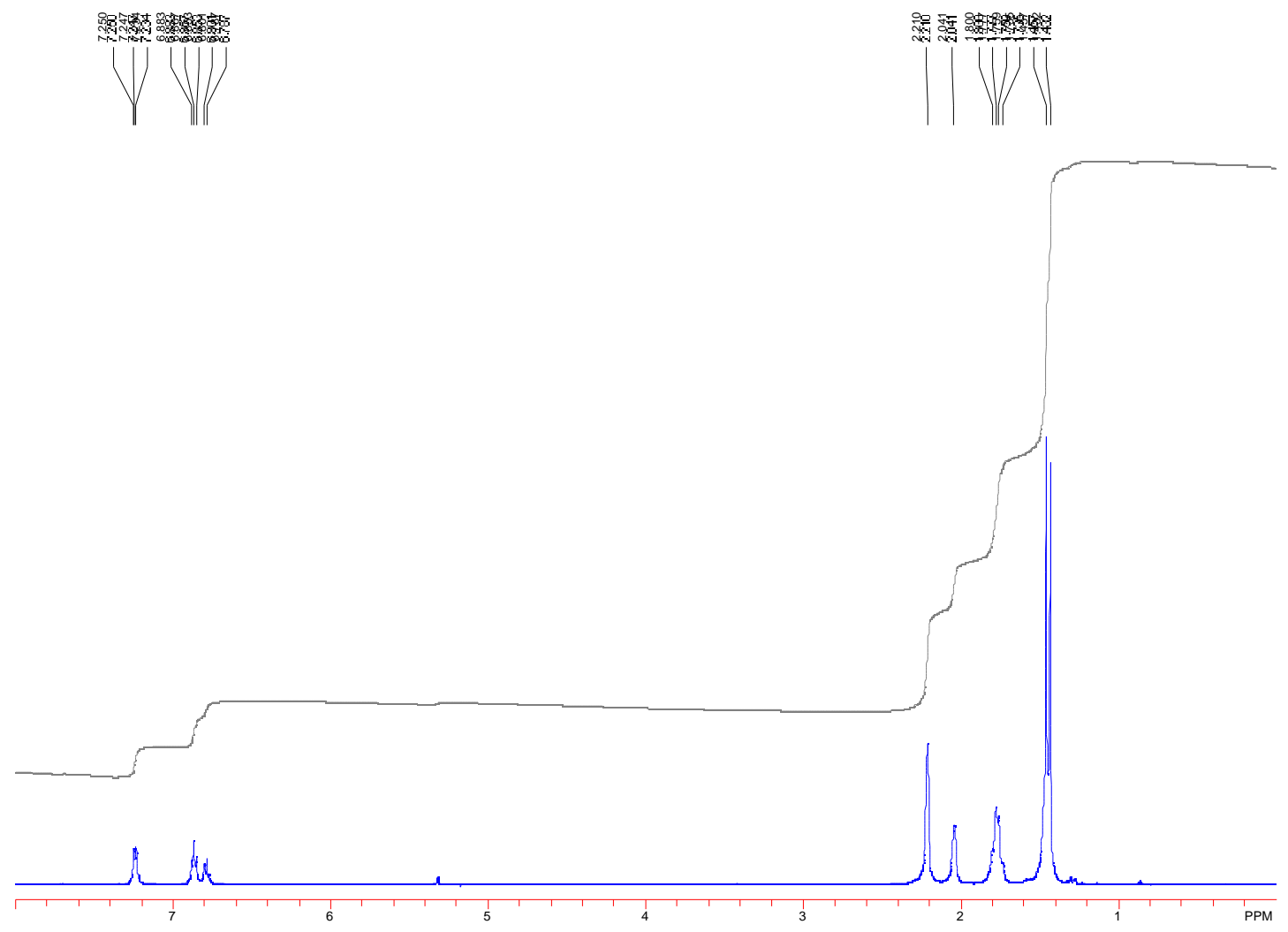

Figure 1. ${ }^{1} \mathrm{H}$ NMR spectrum of $\mathbf{3 a}$. 


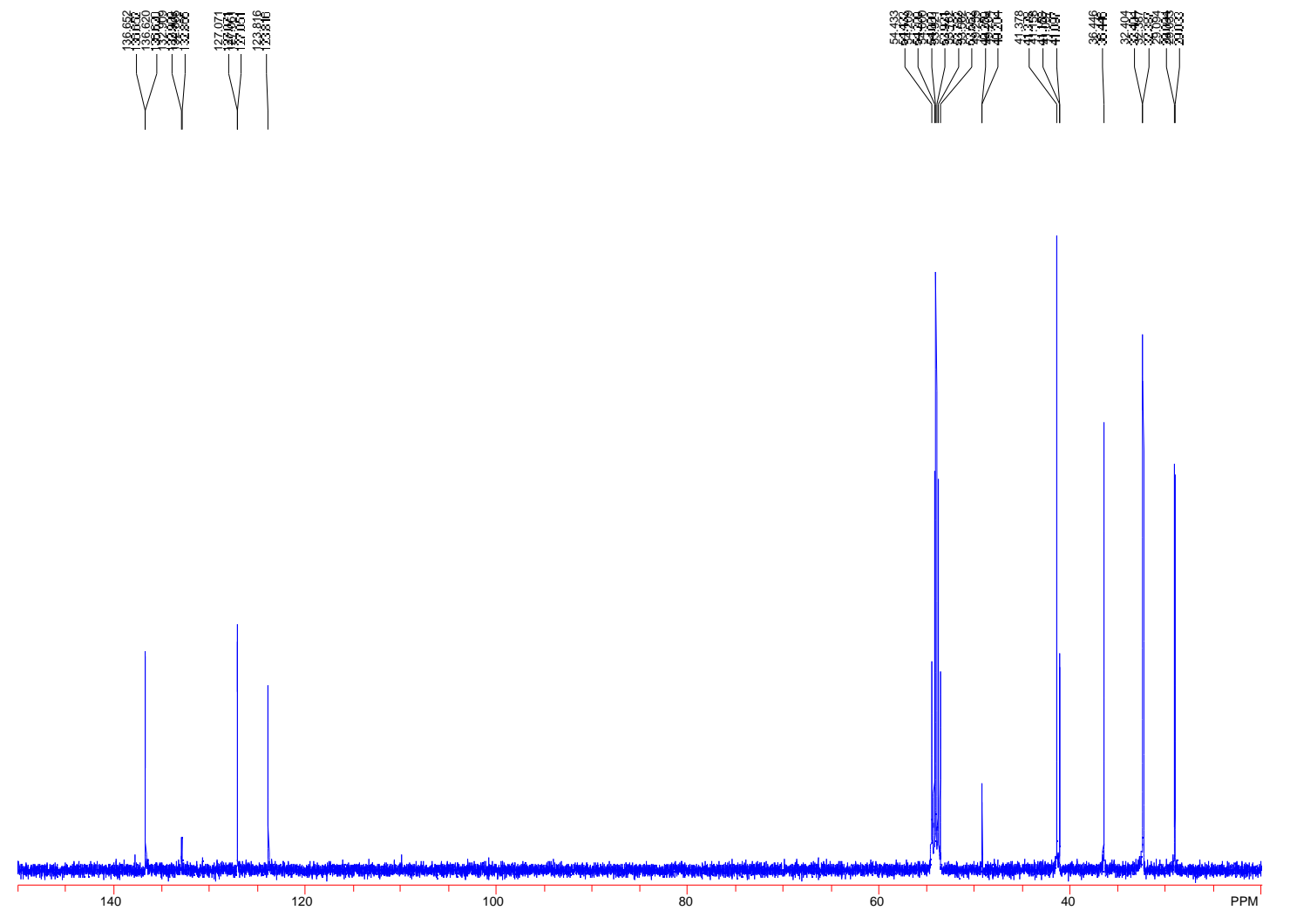

Figure 2. ${ }^{13} \mathrm{C}$ NMR spectrum of $\mathbf{3 a}$. 


\section{X-ray data for $5 a$.}

Table 1. Crystal data and structure refinement for $\mathbf{5 a}$.

Identification code

Empirical formula

Formula weight

Temperature

Wavelength

Crystal system

Space group

Volume

Z

Density (calculated)

Absorption coefficient

$\mathrm{F}(000)$

Crystal size

Theta range for data collection

Index ranges

Reflections collected

Independent reflections

Completeness to theta $=28.27 \infty$

Absorption correction

Refinement method

Data / restraints / parameters

Goodness-of-fit on $\mathrm{F}^{2}$

Final $\mathrm{R}$ indices [I $>2 \operatorname{sigma}(\mathrm{I})]$

$\mathrm{R}$ indices (all data)

Largest diff. peak and hole hart10

C25 H38 F3 O3 P Pd S

612.98

223(2) K

$0.71073 \approx$

Monoclinic

$\mathrm{P} 2(1) / \mathrm{c}$

$\mathrm{a}=9.933(9) \AA \quad \alpha=90^{\circ}$.

$\mathrm{b}=16.926(16) \AA \quad \beta=105.77(2)^{\circ}$.

$\mathrm{c}=16.484(15) \AA \quad \gamma=90^{\circ}$.

2667(4) $\AA^{3}$

4

$1.527 \mathrm{Mg} / \mathrm{m}^{3}$

$0.880 \mathrm{~mm}^{-1}$

1264

$0.30 \times 0.20 \times 0.20 \mathrm{~mm}^{3}$

1.76 to $28.27^{\circ}$.

$-13<=\mathrm{h}<=12,-22<=\mathrm{k}<=22,-21<=\mathrm{l}<=21$

19314

$6350[\mathrm{R}(\mathrm{int})=0.0352]$

$95.9 \%$

\section{SADABS}

Full-matrix least-squares on $\mathrm{F}^{2}$

6350 / 0 / 308

1.156

$\mathrm{R} 1=0.0340, \mathrm{wR} 2=0.0902$

$\mathrm{R} 1=0.0408, \mathrm{wR} 2=0.0933$

0.843 and -0.347 e. $\AA^{-3}$ 
Table 2. Atomic coordinates ( $x 10^{4}$ ) and equivalent isotropic displacement parameters $\left(\AA^{2} \times 10^{3}\right)$ for 5a. U(eq) is defined as one third of the trace of the orthogonalized $U^{i j}$ tensor.

\begin{tabular}{|c|c|c|c|c|}
\hline & $\mathrm{x}$ & $\mathrm{y}$ & $\mathrm{z}$ & $\mathrm{U}(\mathrm{eq})$ \\
\hline $\operatorname{Pd}(1)$ & $9970(1)$ & $1357(1)$ & $906(1)$ & $29(1)$ \\
\hline$S(1)$ & $11690(1)$ & $605(1)$ & $2657(1)$ & $40(1)$ \\
\hline $\mathrm{P}(1)$ & $9015(1)$ & $2392(1)$ & $93(1)$ & $29(1)$ \\
\hline $\mathrm{F}(1)$ & $13975(2)$ & $-120(2)$ & $2606(2)$ & 101(1) \\
\hline $\mathrm{F}(2)$ & $14167(2)$ & $607(2)$ & $3689(2)$ & $129(1)$ \\
\hline $\mathrm{F}(3)$ & $14032(3)$ & $1114(2)$ & $2459(2)$ & $126(1)$ \\
\hline $\mathrm{O}(1)$ & $11242(2)$ & $500(1)$ & $1745(1)$ & $41(1)$ \\
\hline $\mathrm{O}(2)$ & $11366(3)$ & $-35(2)$ & $3116(2)$ & $95(1)$ \\
\hline $\mathrm{O}(3)$ & $11454(3)$ & $1384(1)$ & $2913(2)$ & $80(1)$ \\
\hline $\mathrm{C}(1)$ & $8341(2)$ & $653(1)$ & $722(1)$ & $32(1)$ \\
\hline $\mathrm{C}(2)$ & $8060(3)$ & $106(1)$ & $74(2)$ & $43(1)$ \\
\hline $\mathrm{C}(3)$ & $7043(3)$ & $-467(2)$ & $36(2)$ & $57(1)$ \\
\hline C(4) & $6333(3)$ & $-496(2)$ & $633(2)$ & $63(1)$ \\
\hline$C(5)$ & $6628(3)$ & $36(2)$ & $1288(2)$ & $56(1)$ \\
\hline$C(6)$ & $7627(3)$ & $611(2)$ & $1337(2)$ & $44(1)$ \\
\hline$C(7)$ & $10555(2)$ & $3057(1)$ & $600(1)$ & $28(1)$ \\
\hline $\mathrm{C}(8)$ & $10804(3)$ & $3764(1)$ & $72(2)$ & $38(1)$ \\
\hline $\mathrm{C}(9)$ & $12122(3)$ & $4211(1)$ & $545(2)$ & $46(1)$ \\
\hline $\mathrm{C}(10)$ & $13385(3)$ & $3661(2)$ & $713(2)$ & $57(1)$ \\
\hline $\mathrm{C}(11)$ & 13181(3) & $2980(2)$ & $1277(2)$ & $48(1)$ \\
\hline$C(12)$ & 11861(3) & $2515(1)$ & $811(2)$ & $37(1)$ \\
\hline$C(13)$ & $10430(2)$ & $3372(1)$ & $1460(1)$ & $30(1)$ \\
\hline $\mathrm{C}(14)$ & $11745(3)$ & $3836(2)$ & $1923(2)$ & $41(1)$ \\
\hline$C(15)$ & $11945(3)$ & $4528(1)$ & $1377(2)$ & $46(1)$ \\
\hline$C(16)$ & $13017(3)$ & $3293(2)$ & $2109(2)$ & $49(1)$ \\
\hline $\mathrm{C}(17)$ & $7322(2)$ & $2827(1)$ & $220(2)$ & $38(1)$ \\
\hline $\mathrm{C}(18)$ & $6070(3)$ & $2376(2)$ & $-330(2)$ & $59(1)$ \\
\hline C(19) & $7155(3)$ & $3714(1)$ & $-5(2)$ & $44(1)$ \\
\hline
\end{tabular}




$\begin{array}{lrrrr}\mathrm{C}(20) & 7269(3) & 2724(2) & 1132(2) & 45(1) \\ \mathrm{C}(21) & 8882(3) & 2254(2) & -1068(1) & 51(1) \\ \mathrm{C}(22) & 8340(4) & 2988(2) & -1609(2) & 59(1) \\ \mathrm{C}(23) & 7900(5) & 1549(2) & -1425(2) & 77(1) \\ \mathrm{C}(24) & 10331(4) & 2034(2) & -1167(2) & 66(1) \\ \mathrm{C}(25) & 13577(3) & 551(2) & 2868(2) & 67(1)\end{array}$


Table 3. Bond lengths $[\AA]$ and angles $\left[{ }^{\circ}\right]$ for $\mathbf{5 a}$.

\begin{tabular}{|c|c|}
\hline $\operatorname{Pd}(1)-C(1)$ & $1.967(3)$ \\
\hline $\mathrm{Pd}(1)-\mathrm{H}(12)$ & 2.0653 \\
\hline $\mathrm{Pd}(1)-\mathrm{O}(1)$ & $2.159(2)$ \\
\hline $\mathrm{Pd}(1)-\mathrm{P}(1)$ & $2.2523(16)$ \\
\hline $\mathrm{S}(1)-\mathrm{O}(2)$ & $1.408(2)$ \\
\hline $\mathrm{S}(1)-\mathrm{O}(3)$ & $1.423(2)$ \\
\hline $\mathrm{S}(1)-\mathrm{O}(1)$ & $1.458(2)$ \\
\hline$S(1)-C(25)$ & $1.813(4)$ \\
\hline$P(1)-C(21)$ & $1.897(3)$ \\
\hline$P(1)-C(17)$ & $1.898(3)$ \\
\hline $\mathrm{P}(1)-\mathrm{C}(7)$ & $1.901(3)$ \\
\hline $\mathrm{F}(1)-\mathrm{C}(25)$ & $1.313(4)$ \\
\hline $\mathrm{F}(2)-\mathrm{C}(25)$ & $1.325(4)$ \\
\hline $\mathrm{F}(3)-\mathrm{C}(25)$ & $1.316(4)$ \\
\hline$C(1)-C(2)$ & $1.384(3)$ \\
\hline$C(1)-C(6)$ & $1.387(3)$ \\
\hline$C(2)-C(3)$ & $1.390(4)$ \\
\hline$C(3)-C(4)$ & $1.359(5)$ \\
\hline$C(4)-C(5)$ & $1.374(5)$ \\
\hline$C(5)-C(6)$ & $1.377(4)$ \\
\hline$C(7)-C(8)$ & $1.539(3)$ \\
\hline $\mathrm{C}(7)-\mathrm{C}(12)$ & $1.549(3)$ \\
\hline $\mathrm{C}(7)-\mathrm{C}(13)$ & $1.550(3)$ \\
\hline $\mathrm{C}(8)-\mathrm{C}(9)$ & $1.529(4)$ \\
\hline $\mathrm{C}(9)-\mathrm{C}(10)$ & $1.527(4)$ \\
\hline$C(9)-C(15)$ & $1.527(4)$ \\
\hline $\mathrm{C}(10)-\mathrm{C}(11)$ & $1.527(4)$ \\
\hline $\mathrm{C}(11)-\mathrm{C}(16)$ & $1.520(4)$ \\
\hline $\mathrm{C}(11)-\mathrm{C}(12)$ & $1.543(4)$ \\
\hline $\mathrm{C}(13)-\mathrm{C}(14)$ & $1.537(3)$ \\
\hline $\mathrm{C}(14)-\mathrm{C}(15)$ & $1.523(4)$ \\
\hline$C(14)-C(16)$ & $1.525(4)$ \\
\hline $\mathrm{C}(17)-\mathrm{C}(20)$ & $1.528(4)$ \\
\hline $\mathrm{C}(17)-\mathrm{C}(18)$ & $1.528(4)$ \\
\hline
\end{tabular}




\begin{tabular}{|c|c|}
\hline$C(17)-C(19)$ & $1.544(3)$ \\
\hline $\mathrm{C}(21)-\mathrm{C}(24)$ & $1.538(5)$ \\
\hline $\mathrm{C}(21)-\mathrm{C}(22)$ & $1.539(4)$ \\
\hline $\mathrm{C}(21)-\mathrm{C}(23)$ & $1.551(4)$ \\
\hline $\mathrm{C}(1)-\mathrm{Pd}(1)-\mathrm{H}(12)$ & 176.9 \\
\hline $\mathrm{C}(1)-\mathrm{Pd}(1)-\mathrm{O}(1)$ & $90.29(10)$ \\
\hline $\mathrm{H}(12)-\mathrm{Pd}(1)-\mathrm{O}(1)$ & 87.2 \\
\hline $\mathrm{C}(1)-\mathrm{Pd}(1)-\mathrm{P}(1)$ & $100.58(9)$ \\
\hline $\mathrm{H}(12)-\mathrm{Pd}(1)-\mathrm{P}(1)$ & 81.9 \\
\hline $\mathrm{O}(1)-\mathrm{Pd}(1)-\mathrm{P}(1)$ & $169.12(5)$ \\
\hline $\mathrm{O}(2)-\mathrm{S}(1)-\mathrm{O}(3)$ & $118.29(18)$ \\
\hline $\mathrm{O}(2)-\mathrm{S}(1)-\mathrm{O}(1)$ & $114.27(15)$ \\
\hline $\mathrm{O}(3)-\mathrm{S}(1)-\mathrm{O}(1)$ & $112.88(13)$ \\
\hline $\mathrm{O}(2)-\mathrm{S}(1)-\mathrm{C}(25)$ & $103.50(17)$ \\
\hline $\mathrm{O}(3)-\mathrm{S}(1)-\mathrm{C}(25)$ & $103.57(17)$ \\
\hline $\mathrm{O}(1)-\mathrm{S}(1)-\mathrm{C}(25)$ & $101.62(14)$ \\
\hline $\mathrm{C}(21)-\mathrm{P}(1)-\mathrm{C}(17)$ & $109.76(13)$ \\
\hline $\mathrm{C}(21)-\mathrm{P}(1)-\mathrm{C}(7)$ & $110.12(12)$ \\
\hline $\mathrm{C}(17)-\mathrm{P}(1)-\mathrm{C}(7)$ & $110.70(12)$ \\
\hline $\mathrm{C}(21)-\mathrm{P}(1)-\mathrm{Pd}(1)$ & $113.99(9)$ \\
\hline $\mathrm{C}(17)-\mathrm{P}(1)-\mathrm{Pd}(1)$ & $118.60(8)$ \\
\hline $\mathrm{C}(7)-\mathrm{P}(1)-\mathrm{Pd}(1)$ & $92.31(9)$ \\
\hline $\mathrm{S}(1)-\mathrm{O}(1)-\mathrm{Pd}(1)$ & $122.74(11)$ \\
\hline$C(2)-C(1)-C(6)$ & $119.6(2)$ \\
\hline $\mathrm{C}(2)-\mathrm{C}(1)-\mathrm{Pd}(1)$ & $120.61(18)$ \\
\hline$C(6)-C(1)-P d(1)$ & $118.74(19)$ \\
\hline $\mathrm{C}(1)-\mathrm{C}(2)-\mathrm{C}(3)$ & $119.5(3)$ \\
\hline $\mathrm{C}(4)-\mathrm{C}(3)-\mathrm{C}(2)$ & $120.4(3)$ \\
\hline $\mathrm{C}(3)-\mathrm{C}(4)-\mathrm{C}(5)$ & $120.3(3)$ \\
\hline$C(4)-C(5)-C(6)$ & $120.3(3)$ \\
\hline$C(5)-C(6)-C(1)$ & $119.8(3)$ \\
\hline$C(8)-C(7)-C(12)$ & $109.52(19)$ \\
\hline $\mathrm{C}(8)-\mathrm{C}(7)-\mathrm{C}(13)$ & $108.35(18)$ \\
\hline$C(12)-C(7)-C(13)$ & $105.59(18)$ \\
\hline $\mathrm{C}(8)-\mathrm{C}(7)-\mathrm{P}(1)$ & $116.48(17)$ \\
\hline $\mathrm{C}(12)-\mathrm{C}(7)-\mathrm{P}(1)$ & $105.83(16)$ \\
\hline
\end{tabular}




$\begin{array}{ll}\mathrm{C}(13)-\mathrm{C}(7)-\mathrm{P}(1) & 110.52(14) \\ \mathrm{C}(9)-\mathrm{C}(8)-\mathrm{C}(7) & 110.1(2) \\ \mathrm{C}(10)-\mathrm{C}(9)-\mathrm{C}(15) & 110.0(3) \\ \mathrm{C}(10)-\mathrm{C}(9)-\mathrm{C}(8) & 109.8(2) \\ \mathrm{C}(15)-\mathrm{C}(9)-\mathrm{C}(8) & 109.9(2) \\ \mathrm{C}(9)-\mathrm{C}(10)-\mathrm{C}(11) & 109.4(2) \\ \mathrm{C}(16)-\mathrm{C}(11)-\mathrm{C}(10) & 110.6(2) \\ \mathrm{C}(16)-\mathrm{C}(11)-\mathrm{C}(12) & 109.9(2) \\ \mathrm{C}(10)-\mathrm{C}(11)-\mathrm{C}(12) & 108.5(2) \\ \mathrm{C}(11)-\mathrm{C}(12)-\mathrm{C}(7) & 110.9(2) \\ \mathrm{C}(14)-\mathrm{C}(13)-\mathrm{C}(7) & 111.18(19) \\ \mathrm{C}(15)-\mathrm{C}(14)-\mathrm{C}(16) & 110.4(2) \\ \mathrm{C}(15)-\mathrm{C}(14)-\mathrm{C}(13) & 109.2(2) \\ \mathrm{C}(16)-\mathrm{C}(14)-\mathrm{C}(13) & 109.7(2) \\ \mathrm{C}(14)-\mathrm{C}(15)-\mathrm{C}(9) & 109.0(2) \\ \mathrm{C}(11)-\mathrm{C}(16)-\mathrm{C}(14) & 108.3(2) \\ \mathrm{C}(20)-\mathrm{C}(17)-\mathrm{C}(18) & 106.7(2) \\ \mathrm{C}(20)-\mathrm{C}(17)-\mathrm{C}(19) & 108.5(2) \\ \mathrm{C}(18)-\mathrm{C}(17)-\mathrm{C}(19) & 109.0(2) \\ \mathrm{C}(20)-\mathrm{C}(17)-\mathrm{P}(1) & 109.43(16) \\ \mathrm{C}(18)-\mathrm{C}(17)-\mathrm{P}(1) & 110.1(2) \\ \mathrm{C}(19)-\mathrm{C}(17)-\mathrm{P}(1) & 112.97(17) \\ \mathrm{C}(24)-\mathrm{C}(21)-\mathrm{C}(22) & 108.9(2) \\ \mathrm{C}(24)-\mathrm{C}(21)-\mathrm{C}(23) & 106.5(3) \\ \mathrm{C}(22)-\mathrm{C}(21)-\mathrm{C}(23) & 108.4(3) \\ \mathrm{C}(24)-\mathrm{C}(21)-\mathrm{P}(1) & 109.03(19) \\ \mathrm{C}(22)-\mathrm{C}(21)-\mathrm{P}(1) & 113.75(18) \\ \mathrm{C}(23)-\mathrm{C}(21)-\mathrm{P}(1) & 110.0(2) \\ \mathrm{F}(1)-\mathrm{C}(25)-\mathrm{F}(3) & 106.4(3) \\ \mathrm{F}(1)-\mathrm{C}(25)-\mathrm{F}(2) & 108.0(3) \\ \mathrm{F}(3)-\mathrm{C}(25)-\mathrm{F}(2) & 110.7(3) \\ \mathrm{F}(1)-\mathrm{C}(25)-\mathrm{S}(1) & 111.6(2) \\ \mathrm{F}(3)-\mathrm{C}(25)-\mathrm{S}(1) & 110.3(3) \\ \mathrm{F}(2)-\mathrm{C}(25)-\mathrm{S}(1) & 109.9(3) \\ & \end{array}$

Symmetry transformations used to generate equivalent atoms: 
Table 4. Anisotropic displacement parameters $\left(\AA^{2} \times 10^{3}\right)$ for $\mathbf{5 a}$. The anisotropic displacement factor exponent takes the form: $-2 \pi^{2}\left[h^{2} a^{* 2} U^{11}+\ldots+2 h k a^{*} b^{*} U^{12}\right]$

\begin{tabular}{|c|c|c|c|c|c|c|}
\hline & $\mathrm{U}^{11}$ & $\mathrm{U}^{22}$ & $\mathrm{U}^{33}$ & $\mathrm{U}^{23}$ & $\mathrm{U}^{13}$ & $\mathrm{U}^{12}$ \\
\hline $\operatorname{Pd}(1)$ & $37(1)$ & $19(1)$ & $28(1)$ & $3(1)$ & $6(1)$ & $-2(1)$ \\
\hline$S(1)$ & $41(1)$ & $45(1)$ & $35(1)$ & $8(1)$ & $10(1)$ & $4(1)$ \\
\hline $\mathrm{P}(1)$ & $42(1)$ & $20(1)$ & $23(1)$ & $1(1)$ & $5(1)$ & $-2(1)$ \\
\hline $\mathrm{F}(1)$ & $63(1)$ & $101(2)$ & $126(2)$ & $-17(2)$ & $2(1)$ & 41(1) \\
\hline $\mathrm{F}(2)$ & $84(2)$ & $158(3)$ & $102(2)$ & $-42(2)$ & $-48(1)$ & $9(2)$ \\
\hline $\mathrm{F}(3)$ & $70(2)$ & $112(2)$ & 205(3) & $4(2)$ & $50(2)$ & $-36(2)$ \\
\hline $\mathrm{O}(1)$ & $51(1)$ & $30(1)$ & $36(1)$ & $4(1)$ & $1(1)$ & $9(1)$ \\
\hline $\mathrm{O}(2)$ & $85(2)$ & $114(2)$ & $82(2)$ & $59(2)$ & $16(1)$ & $-20(2)$ \\
\hline $\mathrm{O}(3)$ & 102(2) & $78(2)$ & $56(1)$ & $-18(1)$ & $18(1)$ & $40(1)$ \\
\hline $\mathrm{C}(1)$ & $37(1)$ & $19(1)$ & $37(1)$ & $4(1)$ & $4(1)$ & $-4(1)$ \\
\hline $\mathrm{C}(2)$ & $47(2)$ & $25(1)$ & $51(2)$ & $-3(1)$ & $3(1)$ & $1(1)$ \\
\hline$C(3)$ & $56(2)$ & $28(1)$ & $73(2)$ & $-8(1)$ & $-9(2)$ & $-5(1)$ \\
\hline $\mathrm{C}(4)$ & $44(2)$ & $41(2)$ & $92(2)$ & $18(2)$ & $-2(2)$ & $-14(1)$ \\
\hline$C(5)$ & $47(2)$ & $50(2)$ & $71(2)$ & $25(2)$ & $17(1)$ & $-5(1)$ \\
\hline$C(6)$ & $54(2)$ & $37(1)$ & $40(1)$ & $7(1)$ & $13(1)$ & $-4(1)$ \\
\hline$C(7)$ & $34(1)$ & $20(1)$ & $31(1)$ & $3(1)$ & $12(1)$ & $-1(1)$ \\
\hline$C(8)$ & $52(2)$ & $24(1)$ & $42(1)$ & $5(1)$ & $20(1)$ & $-3(1)$ \\
\hline $\mathrm{C}(9)$ & $52(2)$ & $27(1)$ & $64(2)$ & $10(1)$ & $24(1)$ & $-7(1)$ \\
\hline$C(10)$ & $47(2)$ & $42(2)$ & $90(2)$ & $6(1)$ & $33(2)$ & $-9(1)$ \\
\hline $\mathrm{C}(11)$ & $32(1)$ & $33(1)$ & $80(2)$ & $9(1)$ & $16(1)$ & $-1(1)$ \\
\hline$C(12)$ & $42(1)$ & $24(1)$ & $49(1)$ & $2(1)$ & $20(1)$ & $-1(1)$ \\
\hline $\mathrm{C}(13)$ & $36(1)$ & $26(1)$ & $30(1)$ & $-2(1)$ & $9(1)$ & $-3(1)$ \\
\hline $\mathrm{C}(14)$ & $44(1)$ & $32(1)$ & $42(1)$ & $-4(1)$ & $4(1)$ & $-6(1)$ \\
\hline$C(15)$ & $44(2)$ & $27(1)$ & $62(2)$ & $-3(1)$ & $6(1)$ & $-7(1)$ \\
\hline$C(16)$ & $36(1)$ & $41(2)$ & $63(2)$ & $4(1)$ & $-1(1)$ & $-11(1)$ \\
\hline $\mathrm{C}(17)$ & $35(1)$ & $28(1)$ & $44(1)$ & $5(1)$ & $0(1)$ & $-1(1)$ \\
\hline $\mathrm{C}(18)$ & $45(2)$ & $46(2)$ & $72(2)$ & $1(2)$ & $-7(1)$ & $-10(1)$ \\
\hline C(19) & $42(2)$ & $32(1)$ & $52(2)$ & $9(1)$ & $3(1)$ & $8(1)$ \\
\hline $\mathrm{C}(20)$ & $40(1)$ & $44(2)$ & $55(2)$ & $10(1)$ & $18(1)$ & $4(1)$ \\
\hline
\end{tabular}




\begin{tabular}{lcllccc}
$\mathrm{C}(21)$ & $98(2)$ & $28(1)$ & $22(1)$ & $1(1)$ & $11(1)$ & $1(1)$ \\
$\mathrm{C}(22)$ & $108(3)$ & $36(2)$ & $26(1)$ & $6(1)$ & $7(1)$ & $1(2)$ \\
$\mathrm{C}(23)$ & $147(4)$ & $40(2)$ & $33(2)$ & $-9(1)$ & $4(2)$ & $-17(2)$ \\
$\mathrm{C}(24)$ & $118(3)$ & $51(2)$ & $36(1)$ & $-2(1)$ & $35(2)$ & $17(2)$ \\
$\mathrm{C}(25)$ & $46(2)$ & $61(2)$ & $80(2)$ & $-10(2)$ & $-7(2)$ & $-5(2)$ \\
\hline
\end{tabular}


Table 5. Hydrogen coordinates ( $\left.\mathrm{x} 10^{4}\right)$ and isotropic displacement parameters $\left(\AA^{2} \mathrm{x} 10\right.$ 3 ) for $5 \mathbf{a}$.

\begin{tabular}{|c|c|c|c|c|}
\hline & $\mathrm{x}$ & $\mathrm{y}$ & $\mathrm{z}$ & $\mathrm{U}(\mathrm{eq})$ \\
\hline $\mathrm{H}(2 \mathrm{~A})$ & 8554 & 122 & -339 & 51 \\
\hline $\mathrm{H}(3 \mathrm{~A})$ & 6845 & -838 & -406 & 69 \\
\hline $\mathrm{H}(4 \mathrm{~A})$ & 5637 & -880 & 598 & 75 \\
\hline $\mathrm{H}(5 \mathrm{~A})$ & 6146 & 6 & 1706 & 67 \\
\hline $\mathrm{H}(6 \mathrm{~A})$ & 7825 & 974 & 1785 & 52 \\
\hline $\mathrm{H}(8 \mathrm{~A})$ & 10909 & 3576 & -469 & 45 \\
\hline $\mathrm{H}(8 \mathrm{~B})$ & 9996 & 4120 & -40 & 45 \\
\hline $\mathrm{H}(9 \mathrm{~A})$ & 12273 & 4660 & 194 & 55 \\
\hline $\mathrm{H}(10 \mathrm{~A})$ & 14237 & 3953 & 989 & 69 \\
\hline $\mathrm{H}(10 \mathrm{~B})$ & 13488 & 3452 & 178 & 69 \\
\hline $\mathrm{H}(11 \mathrm{~A})$ & 14004 & 2626 & 1387 & 58 \\
\hline $\mathrm{H}(12)$ & 11715 & 2076 & 1165 & $26(6)$ \\
\hline $\mathrm{H}(12 \mathrm{~A})$ & 11993 & 2294 & 289 & 31 \\
\hline $\mathrm{H}(13 \mathrm{~A})$ & 9609 & 3716 & 1367 & 36 \\
\hline $\mathrm{H}(13 \mathrm{~B})$ & 10297 & 2927 & 1811 & 36 \\
\hline $\mathrm{H}(14 \mathrm{~A})$ & 11621 & 4040 & 2461 & 49 \\
\hline $\mathrm{H}(15 \mathrm{~A})$ & 12775 & 4832 & 1670 & 55 \\
\hline $\mathrm{H}(15 \mathrm{~B})$ & 11131 & 4879 & 1269 & 55 \\
\hline $\mathrm{H}(16 \mathrm{~A})$ & 13858 & 3586 & 2407 & 59 \\
\hline $\mathrm{H}(16 \mathrm{~B})$ & 12889 & 2853 & 2467 & 59 \\
\hline $\mathrm{H}(18 \mathrm{~A})$ & 5211 & 2604 & -262 & 88 \\
\hline $\mathrm{H}(18 \mathrm{~B})$ & 6129 & 1826 & -160 & 88 \\
\hline $\mathrm{H}(18 \mathrm{C})$ & 6077 & 2414 & -916 & 88 \\
\hline $\mathrm{H}(19 \mathrm{~A})$ & 6275 & 3903 & 68 & 66 \\
\hline H(19B) & 7166 & 3787 & -587 & 66 \\
\hline $\mathrm{H}(19 \mathrm{C})$ & 7921 & 4007 & 361 & 66 \\
\hline $\mathrm{H}(20 \mathrm{~A})$ & 6404 & 2946 & 1197 & 68 \\
\hline $\mathrm{H}(20 \mathrm{~B})$ & 8057 & 2995 & 1506 & 68 \\
\hline $\mathrm{H}(20 \mathrm{C})$ & 7312 & 2167 & 1272 & 68 \\
\hline
\end{tabular}




\begin{tabular}{lrrrr}
$\mathrm{H}(22 \mathrm{~A})$ & 8300 & 2877 & -2193 & 88 \\
$\mathrm{H}(22 \mathrm{~B})$ & 8967 & 3428 & -1410 & 88 \\
$\mathrm{H}(22 \mathrm{C})$ & 7412 & 3120 & -1566 & 88 \\
$\mathrm{H}(23 \mathrm{~A})$ & 7842 & 1481 & -2018 & 116 \\
$\mathrm{H}(23 \mathrm{~B})$ & 6975 & 1655 & -1361 & 116 \\
$\mathrm{H}(23 \mathrm{C})$ & 8270 & 1072 & -1120 & 116 \\
$\mathrm{H}(24 \mathrm{~A})$ & 10270 & 1961 & -1759 & 98 \\
$\mathrm{H}(24 \mathrm{~B})$ & 10649 & 1548 & -863 & 98 \\
$\mathrm{H}(24 \mathrm{C})$ & 10989 & 2454 & -940 & 98 \\
& & & & \\
\hline
\end{tabular}




\section{X-Ray Structure Report for $\mathbf{2 b}$}

\section{$\underline{\text { Data Collection }}$}

A yellow-orange block crystal of $\mathrm{C}_{18} \mathrm{H}_{32} \mathrm{BrPPd}$ having approximate dimensions of $0.25 \times 0.25 \times 0.25 \mathrm{~mm}$ was mounted with epoxy cement on the tip of a fine glass fiber. All measurements were made on a Nonius KappaCCD diffractometer with graphite monochromated Mo-K $\alpha$ radiation.

Cell constants and an orientation matrix for data collection corresponded to a primitive monoclinic cell with dimensions:

$$
\begin{array}{lll}
\mathrm{a}= & 8.7046(17) \AA & \alpha=90^{\circ} \\
\mathrm{b}= & 17.837(4) \AA & \beta=93.54(3) \mathrm{o} \\
\mathrm{c}= & 12.781(3) \AA & \gamma=90^{\circ} \\
\mathrm{V}= & 1980.6(7) \AA^{3} &
\end{array}
$$

For $\mathrm{Z}=4$ and F.W. $=465.72$, the calculated density is $1.562 \mathrm{~g} / \mathrm{cm}^{3}$. Based on a statistical analysis of intensity distribution, and the successful solution and refinement of the structure, the space group was determined to be: $P 2_{1} / n$ (\#14)

The data were collected at a temperature of 183(2) $\mathrm{K}$ to a maximum $2 \theta$ value of $54.74 \mathrm{O}$. Three omega scans consisting of 66, 57, and 18 data frames, respectively, were collected with a frame width of $1.8^{\circ}$ and a detector-to-crystal distance, Dx, of $35 \mathrm{~mm}$. Each frame was exposed twice (for the purpose of de-zingering) for a total of 54 seconds. The data frames were processed and scaled using the DENZO software package. ${ }^{1}$

\section{$\underline{\text { Data Reduction }}$}

A total of 11601 reflections were collected of which 4147 were unique and observed $\left(\mathrm{R}_{\mathrm{int}}=0.0826\right)$. The linear absorption coefficient, $\mu$, for Mo-K $\alpha$ radiation is $30.29 \mathrm{~cm}^{-1}$ and _-scan absorption corrections were applied. The data were corrected for Lorentz and polarization effects. 


\section{$\underline{\text { Structure Solution and Refinement }}$}

The structure was solved by direct methods and expanded using Fourier techniques $^{2}$. The non-hydrogen atoms were refined anisotropically and hydrogen atoms were treated as idealized contributions. The final cycle of full-matrix least-squares

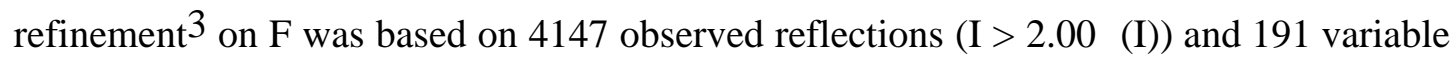
parameters and converged with unweighted and weighted agreement factors of:

$$
\begin{gathered}
\mathrm{R}=\Sigma\|\mathrm{Fo}|-| \mathrm{Fc}\| / \Sigma|\mathrm{Fo}|=0.0574 \\
\mathrm{R}_{\mathrm{W}}=\left[\Sigma \mathrm{w}(|\mathrm{Fo}|-|\mathrm{Fc}|)^{2} / \Sigma \mathrm{w} \mathrm{Fo}^{2}\right]^{1 / 2}=0.1424
\end{gathered}
$$

The maximum and minimum peaks on the final difference Fourier map corresponded to 1.268 and $-0.697 \mathrm{e}^{-} / \AA^{3}$, respectively.

\section{REFERENCES}

(1) Z. Otwinowski and W. Minor, "Processing of X-Ray Diffraction Data Collected in Oscillation Mode," Methods in Enzymology, vol. 276: Macromolecular Crystallography, part A, 307-326, 1997, C.W. Carter, Jr. \& R.M. Sweet, Eds., Academic Press.

(2) Acta Cryst. A46 (1990) 467-473

(3) Least Squares function minimized:

$$
\Sigma w\left(\left|\mathrm{~F}_{\mathrm{O}}\right|-\left|\mathrm{F}_{\mathrm{C}}\right|\right)^{2}
$$




\section{Structural Description}

The compound crystallized in the monoclinic space group $P 2_{1} / n$ with one molecule in the asymmetric unit and four in the unit cell. The geometry about the palladium atom is square planar with the fourth coordination site occupied by a hydrogen atom of $\mathrm{C}(12)$. Although the protons of $\mathrm{C}(12)$ were not located from the electron difference map the positions of $\mathrm{H}(12 \mathrm{~A}-\mathrm{C})$ represent the most probable orientation. This feature is better illustrated in Figure 3. The $\operatorname{Pd}(1)-\mathrm{H}(12 \mathrm{~B})$ distance is $2.18 \AA$ and is comparable to a similar agostic interaction, $2.14 \AA$, in the previously solved JS03. The angles associated with this interaction are $79.2^{\circ}[\mathrm{H}(12 \mathrm{~B})-\mathrm{Pd}(1)-\mathrm{P}(1)], 87.9^{\circ}[\mathrm{H}(12 \mathrm{~B})-$ $\operatorname{Pd}(1)-\mathrm{Br}(1)]$, and $175.4^{\circ}[\mathrm{H}(12 \mathrm{~B})-\mathrm{Pd}(1)-\mathrm{C}(13)]$. The coordination plane of palladium, including $\mathrm{H}(12 \mathrm{~B})$, is planar (mean deviation $=0.050 \AA$ ) and the phenyl ring $[\mathrm{C}(13-18)]$ is offset from this plane by $87.4^{\circ}$. There are no significant intermolecular contacts. 
Table 6. Crystal data and structure refinement for $\mathbf{2 b}$.

Identification code

Empirical formula

Formula weight

Temperature

Wavelength

Crystal system

Space group

Unit cell dimensions

Volume

$\mathrm{Z}$

Density (calculated)

Absorption coefficient

$\mathrm{F}(000)$

Crystal size

Theta range for data collection

Index ranges

Reflections collected

Independent reflections

Completeness to theta $=27.87^{\circ}$

Absorption correction

Max. and min. transmission

Refinement method

Data / restraints / parameters

Goodness-of-fit on $\mathrm{F}^{2}$

Final R indices [I $>2 \operatorname{sigma(I)]~}$

$\mathrm{R}$ indices (all data)

Largest diff. peak and hole hartwig_js04

$\mathrm{C}_{18} \mathrm{H}_{32} \mathrm{Br}$ P Pd

465.72

183(2) K

$0.71073 \AA$

Monoclinic

$\mathrm{P} 2(1) / \mathrm{n}$

$$
\begin{array}{ll}
\mathrm{a}=8.7046(17) \AA & \alpha=90^{\circ} . \\
\mathrm{b}=17.837(4) \AA & \beta=93.54(3)^{\circ} . \\
\mathrm{c}=12.781(3) \AA & \gamma=90^{\circ} .
\end{array}
$$

1980.6(7) $\AA^{3}$

4

$1.562 \mathrm{~g} / \mathrm{cm}^{3}$

$30.29 \mathrm{~cm}^{-1}$

944

$0.25 \times 0.25 \times 0.25 \mathrm{~mm}^{3}$

13.27 to $27.87^{\circ}$.

$-11<=\mathrm{h}<=9,-23<=\mathrm{k}<=23,-11<=\mathrm{l}<=16$

11601

$4147[\mathrm{R}(\mathrm{int})=0.0826]$

$87.8 \%$

psi-scans

0.5181 and 0.5181

Full-matrix least-squares on $\mathrm{F}^{2}$

4147 / 0 / 191

1.093

$\mathrm{R} 1=0.0574, \mathrm{wR} 2=0.1424$

$\mathrm{R} 1=0.0861, \mathrm{wR} 2=0.1555$

1.268 and -0.697 e. $\AA^{-3}$ 
Table 7. Atomic coordinates ( $\mathrm{x} 10^{4}$ ) and equivalent isotropic displacement parameters $\left(\AA^{2} \times 10^{3}\right)$ for $\mathbf{2 b}$. U(eq) is defined as one third of the trace of the orthogonalized $\mathrm{U}^{\mathrm{ij}}$ tensor.

\begin{tabular}{|c|c|c|c|c|}
\hline & $\mathrm{x}$ & $\mathrm{y}$ & $\mathrm{Z}$ & $\mathrm{U}(\mathrm{eq})$ \\
\hline $\operatorname{Pd}(1)$ & $9313(1)$ & $110(1)$ & $7387(1)$ & $33(1)$ \\
\hline $\operatorname{Br}(1)$ & $10113(1)$ & $1427(1)$ & $7318(1)$ & $48(1)$ \\
\hline $\mathrm{P}(1)$ & $9143(1)$ & $-1169(1)$ & $7383(1)$ & $30(1)$ \\
\hline $\mathrm{C}(1)$ & $7871(7)$ & $-1580(3)$ & $6267(4)$ & $41(1)$ \\
\hline$C(2)$ & $8420(20)$ & $-1256(8)$ & $5253(7)$ & $110(5)$ \\
\hline$C(3)$ & $6218(9)$ & $-1368(7)$ & $6395(9)$ & $88(3)$ \\
\hline $\mathrm{C}(4)$ & $7960(10)$ & $-2433(4)$ & $6186(7)$ & $67(2)$ \\
\hline$C(5)$ & $8737(8)$ & $-1638(3)$ & $8672(4)$ & $46(1)$ \\
\hline$C(6)$ & $9486(17)$ & $-1205(6)$ & $9591(7)$ & $93(3)$ \\
\hline$C(7)$ & $9185(11)$ & $-2461(4)$ & $8735(6)$ & $64(2)$ \\
\hline $\mathrm{C}(8)$ & $6986(11)$ & $-1621(5)$ & $8802(7)$ & $72(2)$ \\
\hline $\mathrm{C}(9)$ & $11262(6)$ & $-1307(3)$ & $7089(6)$ & $48(1)$ \\
\hline$C(10)$ & $11753(9)$ & $-2059(5)$ & $6753(10)$ & $80(3)$ \\
\hline $\mathrm{C}(11)$ & $12294(11)$ & $-1206(7)$ & $8178(10)$ & $88(3)$ \\
\hline $\mathrm{C}(12)$ & $11688(11)$ & $-659(6)$ & $6435(8)$ & $76(2)$ \\
\hline$C(13)$ & $7218(6)$ & $368(3)$ & 7784(4) & $36(1)$ \\
\hline $\mathrm{C}(14)$ & $6078(7)$ & $549(3)$ & $7021(5)$ & $45(1)$ \\
\hline$C(15)$ & $4653(7)$ & $784(4)$ & $7311(6)$ & $56(2)$ \\
\hline$C(16)$ & $4336(8)$ & $838(4)$ & $8343(6)$ & $55(2)$ \\
\hline $\mathrm{C}(17)$ & $5483(8)$ & $697(4)$ & $9106(6)$ & $55(2)$ \\
\hline $\mathrm{C}(18)$ & $6914(8)$ & $451(3)$ & $8827(5)$ & $46(1)$ \\
\hline
\end{tabular}


Table 8. Bond lengths $[\AA]$ and angles $\left[^{\circ}\right]$ for $\mathbf{2 b}$.

\begin{tabular}{|c|c|c|c|}
\hline $\operatorname{Pd}(1)-C(13)$ & $1.977(5)$ & $C(2)-C(1)-P(1)$ & $107.1(5)$ \\
\hline $\mathrm{Pd}(1)-\mathrm{P}(1)$ & $2.2854(13)$ & $\mathrm{C}(4)-\mathrm{C}(1)-\mathrm{P}(1)$ & $114.0(4)$ \\
\hline $\operatorname{Pd}(1)-\operatorname{Br}(1)$ & $2.4537(8)$ & $C(7)-C(5)-C(6)$ & $110.7(7)$ \\
\hline $\mathrm{P}(1)-\mathrm{C}(1)$ & $1.898(5)$ & $C(7)-C(5)-C(8)$ & $105.4(6)$ \\
\hline $\mathrm{P}(1)-\mathrm{C}(5)$ & $1.902(6)$ & $C(6)-C(5)-C(8)$ & $106.6(8)$ \\
\hline$P(1)-C(9)$ & $1.921(6)$ & $\mathrm{C}(7)-\mathrm{C}(5)-\mathrm{P}(1)$ & $114.2(5)$ \\
\hline$C(1)-C(3)$ & $1.507(11)$ & $C(6)-C(5)-P(1)$ & $110.6(5)$ \\
\hline $\mathrm{C}(1)-\mathrm{C}(2)$ & $1.521(14)$ & $\mathrm{C}(8)-\mathrm{C}(5)-\mathrm{P}(1)$ & $108.8(4)$ \\
\hline $\mathrm{C}(1)-\mathrm{C}(4)$ & $1.527(10)$ & $C(10)-C(9)-C(12)$ & $117.1(7)$ \\
\hline$C(5)-C(7)$ & $1.519(10)$ & $\mathrm{C}(10)-\mathrm{C}(9)-\mathrm{C}(11)$ & $101.3(8)$ \\
\hline$C(5)-C(6)$ & $1.519(12)$ & $\mathrm{C}(12)-\mathrm{C}(9)-\mathrm{C}(11)$ & $104.6(7)$ \\
\hline$C(5)-C(8)$ & $1.543(12)$ & $\mathrm{C}(10)-\mathrm{C}(9)-\mathrm{P}(1)$ & $118.1(5)$ \\
\hline $\mathrm{C}(9)-\mathrm{C}(10)$ & $1.479(11)$ & $\mathrm{C}(12)-\mathrm{C}(9)-\mathrm{P}(1)$ & $106.6(5)$ \\
\hline $\mathrm{C}(9)-\mathrm{C}(12)$ & $1.488(13)$ & $\mathrm{C}(11)-\mathrm{C}(9)-\mathrm{P}(1)$ & $107.8(6)$ \\
\hline $\mathrm{C}(9)-\mathrm{C}(11)$ & $1.620(14)$ & $\mathrm{C}(18)-\mathrm{C}(13)-\mathrm{C}(14)$ & $118.9(5)$ \\
\hline $\mathrm{C}(13)-\mathrm{C}(18)$ & $1.383(9)$ & $C(18)-C(13)-P d(1)$ & $120.4(4)$ \\
\hline $\mathrm{C}(13)-\mathrm{C}(14)$ & $1.386(8)$ & $\mathrm{C}(14)-\mathrm{C}(13)-\mathrm{Pd}(1)$ & $120.3(4)$ \\
\hline $\mathrm{C}(14)-\mathrm{C}(15)$ & $1.382(10)$ & $C(15)-C(14)-C(13)$ & $119.9(6)$ \\
\hline$C(15)-C(16)$ & $1.368(12)$ & $C(16)-C(15)-C(14)$ & 121.1(6) \\
\hline$C(16)-C(17)$ & $1.375(12)$ & $C(15)-C(16)-C(17)$ & $119.4(6)$ \\
\hline $\mathrm{C}(17)-\mathrm{C}(18)$ & $1.389(10)$ & $C(16)-C(17)-C(18)$ & $120.0(6)$ \\
\hline $\mathrm{C}(13)-\mathrm{Pd}(1)-\mathrm{P}(1)$ & $99.92(15)$ & $\mathrm{C}(13)-\mathrm{C}(18)-\mathrm{C}(17)$ & $120.6(6)$ \\
\hline$C(13)-\operatorname{Pd}(1)-\operatorname{Br}(1)$ & $93.14(15)$ & & \\
\hline $\mathrm{P}(1)-\mathrm{Pd}(1)-\mathrm{Br}(1)$ & $166.88(4)$ & \multirow{2}{*}{\multicolumn{2}{|c|}{$\begin{array}{l}\text { Symmetry transformations used to } \\
\text { generate equivalent atoms }\end{array}$}} \\
\hline $\mathrm{C}(1)-\mathrm{P}(1)-\mathrm{C}(5)$ & $110.5(3)$ & & \\
\hline $\mathrm{C}(1)-\mathrm{P}(1)-\mathrm{C}(9)$ & $109.0(3)$ & & \\
\hline C(5)-P(1)-C(9) & $110.2(3)$ & & \\
\hline $\mathrm{C}(1)-\mathrm{P}(1)-\mathrm{Pd}(1)$ & $115.00(19)$ & & \\
\hline $\mathrm{C}(5)-\mathrm{P}(1)-\mathrm{Pd}(1)$ & $116.9(2)$ & & \\
\hline $\mathrm{C}(9)-\mathrm{P}(1)-\mathrm{Pd}(1)$ & $93.79(19)$ & & \\
\hline $\mathrm{C}(3)-\mathrm{C}(1)-\mathrm{C}(2)$ & $110.3(9)$ & & \\
\hline$C(3)-C(1)-C(4)$ & $108.1(7)$ & & \\
\hline $\mathrm{C}(2)-\mathrm{C}(1)-\mathrm{C}(4)$ & $107.5(8)$ & & \\
\hline $\mathrm{C}(3)-\mathrm{C}(1)-\mathrm{P}(1)$ & $109.7(4)$ & & \\
\hline
\end{tabular}


Table 9. Anisotropic displacement parameters $\left(\AA^{2} \times 10^{3}\right)$ for $\mathbf{2 b}$. The anisotropic displacement factor exponent takes the form: $-2 \pi^{2}\left[h^{2} a^{* 2} U^{11}+\ldots+2 h k a^{*} b^{*} U^{12}\right]$

\begin{tabular}{|c|c|c|c|c|c|c|}
\hline & $\mathrm{U}^{11}$ & $\mathrm{U}^{22}$ & $\mathrm{U}^{33}$ & $\mathrm{U}^{23}$ & $\mathrm{U}^{13}$ & $\mathrm{U}^{12}$ \\
\hline $\operatorname{Pd}(1)$ & $31(1)$ & $25(1)$ & $44(1)$ & $-1(1)$ & $5(1)$ & $1(1)$ \\
\hline $\operatorname{Br}(1)$ & $47(1)$ & $28(1)$ & $70(1)$ & $-1(1)$ & $15(1)$ & $-2(1)$ \\
\hline $\mathrm{P}(1)$ & $27(1)$ & $25(1)$ & $38(1)$ & $-3(1)$ & $2(1)$ & $1(1)$ \\
\hline $\mathrm{C}(1)$ & $40(3)$ & $42(3)$ & $40(3)$ & $-7(2)$ & $-5(2)$ & $-7(2)$ \\
\hline $\mathrm{C}(2)$ & $169(12)$ & $110(7)$ & $47(4)$ & $7(5)$ & $-28(5)$ & $-61(8)$ \\
\hline$C(3)$ & $39(4)$ & $106(7)$ & $116(7)$ & $-60(6)$ & $-24(4)$ & $15(4)$ \\
\hline C(4) & 61(4) & $52(4)$ & $86(5)$ & $-30(4)$ & $-16(3)$ & $5(3)$ \\
\hline$C(5)$ & $64(4)$ & $38(3)$ & $36(3)$ & $10(2)$ & $4(2)$ & $0(3)$ \\
\hline$C(6)$ & $143(10)$ & $80(5)$ & $51(4)$ & $14(4)$ & $-28(5)$ & $-23(6)$ \\
\hline$C(7)$ & $80(5)$ & $44(3)$ & $71(4)$ & $16(3)$ & $23(3)$ & $17(3)$ \\
\hline $\mathrm{C}(8)$ & $81(5)$ & $69(5)$ & $70(4)$ & 21(4) & $36(4)$ & $13(4)$ \\
\hline $\mathrm{C}(9)$ & $27(2)$ & $31(3)$ & $85(4)$ & $-7(3)$ & $12(3)$ & $-1(2)$ \\
\hline$C(10)$ & $41(3)$ & $46(4)$ & $156(8)$ & $-12(4)$ & $35(4)$ & $4(3)$ \\
\hline $\mathrm{C}(11)$ & $57(5)$ & $90(6)$ & 117(7) & $5(5)$ & $0(5)$ & $-10(5)$ \\
\hline $\mathrm{C}(12)$ & $65(5)$ & $68(5)$ & $96(6)$ & $-3(4)$ & $25(4)$ & $-6(4)$ \\
\hline$C(13)$ & $32(2)$ & $27(2)$ & $49(3)$ & $1(2)$ & $3(2)$ & $2(2)$ \\
\hline$C(14)$ & $40(3)$ & $43(3)$ & $50(3)$ & $5(2)$ & $-4(2)$ & $5(2)$ \\
\hline$C(15)$ & $38(3)$ & $38(3)$ & $92(5)$ & $9(3)$ & $-11(3)$ & $4(3)$ \\
\hline$C(16)$ & $45(3)$ & $39(3)$ & $83(5)$ & $2(3)$ & $20(3)$ & $1(3)$ \\
\hline$C(17)$ & $55(4)$ & $46(3)$ & $66(4)$ & $-2(3)$ & $23(3)$ & $3(3)$ \\
\hline $\mathrm{C}(18)$ & $45(3)$ & $41(3)$ & $52(3)$ & $-10(2)$ & $5(2)$ & $2(2)$ \\
\hline
\end{tabular}


Table 10. Hydrogen coordinates ( $\left.x 10^{4}\right)$ and isotropic displacement parameters $\left(\AA^{2} \mathrm{x}\right.$ $10^{3}$ ) for $\mathbf{2 b .}$

\begin{tabular}{|c|c|c|c|c|}
\hline & $\mathrm{x}$ & $\mathrm{y}$ & $\mathrm{z}$ & $\mathrm{U}(\mathrm{eq})$ \\
\hline $\mathrm{H}(2 \mathrm{~A})$ & 7783 & -1457 & 4657 & 165 \\
\hline $\mathrm{H}(2 \mathrm{~B})$ & 9495 & -1394 & 5184 & 165 \\
\hline $\mathrm{H}(2 \mathrm{C})$ & 8321 & -709 & 5266 & 165 \\
\hline $\mathrm{H}(3 \mathrm{~A})$ & 5565 & -1582 & 5819 & 132 \\
\hline $\mathrm{H}(3 \mathrm{~B})$ & 6119 & -820 & 6386 & 132 \\
\hline $\mathrm{H}(3 \mathrm{C})$ & 5895 & -1562 & 7064 & 132 \\
\hline $\mathrm{H}(4 \mathrm{~A})$ & 7273 & -2606 & 5598 & 101 \\
\hline $\mathrm{H}(4 \mathrm{~B})$ & 7644 & -2659 & 6838 & 101 \\
\hline $\mathrm{H}(4 \mathrm{C})$ & 9019 & -2583 & 6070 & 101 \\
\hline $\mathrm{H}(6 \mathrm{~A})$ & 9272 & -1457 & 10248 & 139 \\
\hline $\mathrm{H}(6 \mathrm{~B})$ & 9068 & -695 & 9593 & 139 \\
\hline $\mathrm{H}(6 \mathrm{C})$ & 10601 & -1183 & 9525 & 139 \\
\hline $\mathrm{H}(7 \mathrm{~A})$ & 8931 & -2664 & 9415 & 96 \\
\hline $\mathrm{H}(7 \mathrm{~B})$ & 10293 & -2510 & 8658 & 96 \\
\hline $\mathrm{H}(7 \mathrm{C})$ & 8619 & -2738 & 8172 & 96 \\
\hline $\mathrm{H}(8 \mathrm{~A})$ & 6762 & -1871 & 9459 & 108 \\
\hline $\mathrm{H}(8 \mathrm{~B})$ & 6450 & -1881 & 8212 & 108 \\
\hline $\mathrm{H}(8 \mathrm{C})$ & 6634 & -1099 & 8820 & 108 \\
\hline $\mathrm{H}(10 \mathrm{~A})$ & 12855 & -2050 & 6634 & 120 \\
\hline $\mathrm{H}(10 \mathrm{~B})$ & 11170 & -2199 & 6102 & 120 \\
\hline $\mathrm{H}(10 \mathrm{C})$ & 11561 & -2426 & 7300 & 120 \\
\hline $\mathrm{H}(11 \mathrm{~A})$ & 13384 & -1274 & 8049 & 133 \\
\hline $\mathrm{H}(11 \mathrm{~B})$ & 11987 & -1580 & 8686 & 133 \\
\hline $\mathrm{H}(11 \mathrm{C})$ & 12137 & -702 & 8458 & 133 \\
\hline $\mathrm{H}(12 \mathrm{~A})$ & 12767 & -705 & 6267 & 113 \\
\hline $\mathrm{H}(12 \mathrm{~B})$ & 11547 & -192 & 6822 & 113 \\
\hline $\mathrm{H}(12 \mathrm{C})$ & 11030 & -652 & 5784 & 113 \\
\hline $\mathrm{H}(14 \mathrm{~A})$ & 6277 & 511 & 6300 & 53 \\
\hline $\mathrm{H}(15 \mathrm{~A})$ & 3879 & 912 & 6784 & 68 \\
\hline $\mathrm{H}(16 \mathrm{~A})$ & 3333 & 970 & 8531 & 66 \\
\hline
\end{tabular}


H(17A)

H(18A)
5296

7693
769

338
9824

9358
66

55 


\section{X-Ray Structure Report for $\mathbf{2 d}$.}

\section{Data Collection}

A red-orange blade crystal of $\mathrm{C}_{54} \mathrm{H}_{52} \mathrm{BrFePPd}$ having approximate dimensions of $0.15 \times 0.15 \times 0.10 \mathrm{~mm}$ was mounted with epoxy cement on the tip of a fine glass fiber. All measurements were made on a Nonius KappaCCD diffractometer with graphite monochromated Mo-K $\alpha$ radiation.

Cell constants and an orientation matrix for data collection corresponded to a primitive monoclinic cell with dimensions:

$$
\begin{array}{lll}
\mathrm{a}=15.380(3) \AA & \alpha=90^{\circ} \\
\mathrm{b}=18.945(4) \AA & \beta=116.14(3)^{\circ} \\
\mathrm{c}=17.221(3) \AA & \gamma=90^{\circ} \\
\mathrm{V}=4504.4(16) \AA^{3} &
\end{array}
$$

For $\mathrm{Z}=4$ and F.W. $=974.09$, the calculated density is $1.436 \mathrm{~g} / \mathrm{cm}^{3}$. Based on a statistical analysis of intensity distribution, and the successful solution and refinement of the structure, the space group was determined to be: $P 2_{1} / n$ (\#14)

The data were collected at a temperature of 183(2) $\mathrm{K}$ to a maximum $2 \theta$ value of $56.62{ }^{\circ}$. Five omega scans consisting of 54, 43, 48, 43, and 14 data frames, respectively, were collected with a frame width of $1.4^{\circ}$ and a detector-to-crystal distance, Dx, of 35 $\mathrm{mm}$. Each frame was exposed twice (for the purpose of de-zingering) for a total of 203 seconds. The data frames were processed and scaled using the DENZO software package. ${ }^{1}$

Data Reduction

A total of 19779 reflections were collected of which 11161 were unique and observed $\left(\mathrm{R}_{\mathrm{int}}=0.0651\right)$. The linear absorption coefficient, $\mu$, for Mo-K $\alpha$ radiation is $16.80 \mathrm{~cm}^{-1}$ and no absorption correction was applied. The data were corrected for Lorentz and polarization effects.

\section{Structure Solution and Refinement}

The structure was solved by direct methods and expanded using Fourier techniques $^{2}$. The non-hydrogen atoms were refined anisotropically and hydrogen atoms were treated as idealized contributions. The final cycle of full-matrix least-squares refinement ${ }^{3}$ on $\mathrm{F}$ was based on 11161 observed reflections $(\mathrm{I}>2.00 \sigma(\mathrm{I})$ ) and 523 variable parameters and converged with unweighted and weighted agreement factors of:

$$
\mathrm{R}=\Sigma\|\mathrm{Fo}|-| \mathrm{Fc}\| / \Sigma|\mathrm{Fo}|=0.0531
$$




$$
\mathrm{R}_{\mathrm{W}}=\left\{\Sigma\left[\mathrm{w}\left(\mathrm{F}_{\mathrm{o}}^{2}-\mathrm{F}_{\mathrm{c}}^{2}\right)^{2}\right] / \Sigma\left[\mathrm{w}\left(\mathrm{F}_{\mathrm{o}}^{2}\right)^{2}\right]\right\}^{1 / 2}=0.0981
$$

The maximum and minimum peaks on the final difference Fourier map corresponded to 0.717 and $-0.724 \mathrm{e}^{-} / \AA^{3}$, respectively.

\section{REFERENCES}

(1) Z. Otwinowski and W. Minor, "Processing of X-Ray Diffraction Data Collected in Oscillation Mode," Methods in Enzymology, vol. 276: Macromolecular Crystallography, part A, 307-326, 1997, C.W. Carter, Jr. \& R.M. Sweet, Eds., Academic Press.

(2) Acta Cryst. A46 (1990) 467-473

(3) Least Squares function minimized:

$$
\Sigma w\left(\mathrm{~F}_{\mathrm{o}}^{2}-\mathrm{F}_{\mathrm{c}}^{2}\right)^{2}
$$




\section{Structural Description}

The compound crystallized in the monoclinic space group $P 2_{1} / n$ with one molecule in the asymmetric unit and four molecules in the unit cell. The geometry about $\operatorname{Pd}(1)$ is square planar with the final coordination site filled by an agostically bound hydrogen, $\mathrm{H}(46 \mathrm{C})$, of a neighboring ${ }^{\mathrm{B}} \mathrm{Bu}$ group. Although refined in idealize locations the hydrogen atoms of $\mathrm{C}(46)$ were initially located from the electron difference map. The experimental coordinates compared well with the calculated coordinates and the atoms were idealized for ease of refinement. The $\operatorname{Pd}(1)-\mathrm{H}(46 \mathrm{C})$ distance is $2.13 \AA$ and, more significantly, the $\operatorname{Pd}(1)-\mathrm{C}(46)$ distance is $\sim 2.78 \AA$. The coordination plane of $\operatorname{Pd}(1)$ possesses a mean deviation of $0.077 \AA$ and the coordinated phenyl ring, C(49-54), is offset by $98.3^{\circ}$. This phenyl ring is skewed slightly from the $\mathrm{H}(46 \mathrm{C})-\mathrm{Pd}(1)-\mathrm{C}(49)$ vector by $17.3^{\circ}$ and is a direct effect of the steric demands of the phosphine ligand. This feature is better illustrated in Figures $\mathbf{3}$ and $\mathbf{4}$.

The five phenyl rings of the substituted ferrocene are oriented in a "paddlewheel" geometry with offsetting angles between the rings and the central $\mathrm{Cp}$ ring ranging from $44.0-55.5^{\circ}$. $\mathrm{Fe}(1)$ is equidistant from both $\mathrm{Cp}$-centroids, $1.67 \AA$, and $\mathrm{Fe}-\mathrm{C}$ distances range from 2.049(4) - 2.098(3) $\AA$ for $\mathrm{C}(2-10)$. Phosphine substitution in the top $\mathrm{Cp}$ ring results in an elongated $\mathrm{Fe}(1)-\mathrm{C}(1)$ distance of 2.125(3) $\AA$. The two Cp rings are offset from one another by $16.0^{\circ}$ producing a Cent-Fe-Cent angle of $190.2^{\circ}$. This large angle is directed towards $\operatorname{Pd}(1)$ and the $\mathrm{Fe}-\mathrm{Pd}$ separation is $\sim 4.91 \AA$.

There are no significant intermolecular contacts. 
Table 11. Crystal data and structure refinement for $\mathbf{2 d}$.

Identification code

Empirical formula

Formula weight

Temperature

Wavelength

Crystal system

Space group

Unit cell dimensions

Volume

$\mathrm{Z}$

Density (calculated)

Absorption coefficient

$\mathrm{F}(000)$

Crystal size

Theta range for data collection

Index ranges

Reflections collected

Independent reflections

Completeness to theta $=28.31^{\circ}$

Absorption correction

Max. and min. transmission

Refinement method

Data / restraints / parameters

Goodness-of-fit on $\mathrm{F}^{2}$

Final R indices [I $>2 \operatorname{sigma(I)]~}$

$\mathrm{R}$ indices (all data)

Largest diff. peak and hole hartwig_js05

$\mathrm{C}_{54} \mathrm{H}_{52} \mathrm{Br} \mathrm{Fe} \mathrm{P} \mathrm{Pd}$

974.09

183(2) K

$0.71073 \AA$

Monoclinic

$\mathrm{P} 2(1) / \mathrm{n}$

$$
\begin{array}{ll}
\mathrm{a}=15.380(3) \AA & \alpha=90^{\circ} . \\
\mathrm{b}=18.945(4) \AA & \beta=116.14(3)^{\circ} . \\
\mathrm{c}=17.221(3) \AA & \gamma=90^{\circ} .
\end{array}
$$

4504.4(16) $\AA^{3}$

4

$1.436 \mathrm{~g} / \mathrm{cm}^{3}$

$16.80 \mathrm{~cm}^{-1}$

1992

$0.15 \times 0.15 \times 0.10 \mathrm{~mm}^{3}$

2.15 to $28.31^{\circ}$.

$-20<=\mathrm{h}<=20,-25<=\mathrm{k}<=22,-22<=\mathrm{l}<=22$

19779

$11161[\mathrm{R}(\mathrm{int})=0.0651]$

$99.4 \%$

None

0.8500 and 0.7867

Full-matrix least-squares on $\mathrm{F}^{2}$

11161 / 0 / 523

1.031

$\mathrm{R} 1=0.0531, \mathrm{wR} 2=0.0981$

$\mathrm{R} 1=0.1103, \mathrm{wR} 2=0.1135$

0.717 and -0.724 e. $\AA^{-3}$ 
Table 12. Atomic coordinates ( $x 10^{4}$ ) and equivalent isotropic displacement parameters $\left(\AA^{2} \times 10^{3}\right)$ for $2 \mathbf{d}$. $U(e q)$ is defined as one third of the trace of the orthogonalized $\mathrm{Uij}^{\mathrm{ij}}$ tensor.

\begin{tabular}{|c|c|c|c|c|}
\hline & $\mathrm{x}$ & $\mathrm{y}$ & $\mathrm{z}$ & $\mathrm{U}(\mathrm{eq})$ \\
\hline $\operatorname{Pd}(1)$ & 1999(1) & $5807(1)$ & $7606(1)$ & $31(1)$ \\
\hline $\operatorname{Br}(1)$ & $1117(1)$ & $6904(1)$ & $7070(1)$ & $41(1)$ \\
\hline $\mathrm{Fe}(1)$ & $4387(1)$ & $4386(1)$ & $7125(1)$ & $24(1)$ \\
\hline $\mathrm{P}(1)$ & $2465(1)$ & $4671(1)$ & 7911(1) & $29(1)$ \\
\hline $\mathrm{C}(1)$ & $3532(3)$ & $4378(2)$ & $7814(2)$ & $25(1)$ \\
\hline$C(2)$ & $3780(3)$ & $3676(2)$ & $7672(2)$ & $29(1)$ \\
\hline$C(3)$ & $4801(3)$ & $3612(2)$ & $8055(2)$ & $30(1)$ \\
\hline $\mathrm{C}(4)$ & $5204(3)$ & $4277(2)$ & $8433(2)$ & $29(1)$ \\
\hline$C(5)$ & $4431(3)$ & $4749(2)$ & $8267(2)$ & $29(1)$ \\
\hline$C(6)$ & $3940(2)$ & 4993(2) & $6011(2)$ & $26(1)$ \\
\hline$C(7)$ & $3927(2)$ & $4257(2)$ & $5792(2)$ & $25(1)$ \\
\hline$C(8)$ & $4888(3)$ & $3981(2)$ & $6287(2)$ & $28(1)$ \\
\hline $\mathrm{C}(9)$ & $5494(3)$ & $4545(2)$ & $6788(2)$ & $28(1)$ \\
\hline$C(10)$ & 4911(3) & $5173(2)$ & $6628(2)$ & $26(1)$ \\
\hline $\mathrm{C}(11)$ & $3147(3)$ & $5504(2)$ & $5564(2)$ & $28(1)$ \\
\hline$C(12)$ & $2826(3)$ & $5979(2)$ & $5998(2)$ & $35(1)$ \\
\hline$C(13)$ & $2136(3)$ & $6490(2)$ & $5552(3)$ & $43(1)$ \\
\hline $\mathrm{C}(14)$ & $1764(3)$ & $6531(2)$ & $4674(3)$ & $41(1)$ \\
\hline$C(15)$ & $2059(3)$ & $6058(2)$ & $4225(2)$ & $38(1)$ \\
\hline$C(16)$ & $2740(3)$ & $5548(2)$ & $4667(2)$ & $31(1)$ \\
\hline $\mathrm{C}(17)$ & $3111(3)$ & $3893(2)$ & $5072(2)$ & $28(1)$ \\
\hline $\mathrm{C}(18)$ & $3286(3)$ & $3527(2)$ & $4456(2)$ & $33(1)$ \\
\hline C(19) & $2515(3)$ & $3252(2)$ & $3720(2)$ & $44(1)$ \\
\hline$C(20)$ & $1583(3)$ & $3337(2)$ & $3604(2)$ & $44(1)$ \\
\hline $\mathrm{C}(21)$ & $1407(3)$ & $3693(2)$ & $4223(2)$ & $42(1)$ \\
\hline $\mathrm{C}(22)$ & $2165(3)$ & $3967(2)$ & $4945(2)$ & $35(1)$ \\
\hline $\mathrm{C}(23)$ & $5191(3)$ & $3249(2)$ & $6205(2)$ & $27(1)$ \\
\hline $\mathrm{C}(24)$ & $5949(3)$ & $3149(2)$ & $5993(2)$ & $37(1)$ \\
\hline
\end{tabular}




\begin{tabular}{|c|c|c|c|c|}
\hline$C(25)$ & $6219(3)$ & $2476(2)$ & $5861(3)$ & $49(1)$ \\
\hline$C(26)$ & $5732(3)$ & 1899(2) & $5954(3)$ & $53(1)$ \\
\hline $\mathrm{C}(27)$ & $4973(3)$ & $1985(2)$ & $6165(3)$ & $46(1)$ \\
\hline $\mathrm{C}(28)$ & $4697(3)$ & $2656(2)$ & $6286(2)$ & $34(1)$ \\
\hline $\mathrm{C}(29)$ & $6566(3)$ & $4503(2)$ & $7341(2)$ & $30(1)$ \\
\hline $\mathrm{C}(30)$ & $7170(3)$ & $5023(2)$ & $7288(2)$ & $36(1)$ \\
\hline $\mathrm{C}(31)$ & $8163(3)$ & 4984(3) & $7765(3)$ & $49(1)$ \\
\hline $\mathrm{C}(32)$ & $8578(3)$ & $4424(3)$ & $8308(3)$ & $56(1)$ \\
\hline$C(33)$ & $7982(3)$ & $3890(2)$ & $8368(3)$ & $51(1)$ \\
\hline C(34) & $6989(3)$ & $3926(2)$ & $7886(2)$ & $37(1)$ \\
\hline $\mathrm{C}(35)$ & $5245(3)$ & $5899(2)$ & $6970(2)$ & $29(1)$ \\
\hline$C(36)$ & $5845(3)$ & $6017(2)$ & $7848(2)$ & $36(1)$ \\
\hline $\mathrm{C}(37)$ & $6175(3)$ & $6686(2)$ & $8144(3)$ & $44(1)$ \\
\hline $\mathrm{C}(38)$ & $5917(3)$ & $7257(2)$ & $7579(3)$ & $49(1)$ \\
\hline C(39) & $5319(3)$ & $7145(2)$ & $6720(3)$ & $46(1)$ \\
\hline$C(40)$ & 4990(3) & $6473(2)$ & $6413(2)$ & $34(1)$ \\
\hline$C(41)$ & $2681(3)$ & $4290(2)$ & $8996(2)$ & $36(1)$ \\
\hline$C(42)$ & $3706(3)$ & $4504(2)$ & $9656(2)$ & $43(1)$ \\
\hline$C(43)$ & 2611(3) & $3478(2)$ & $8972(3)$ & $49(1)$ \\
\hline$C(44)$ & $1958(3)$ & $4605(2)$ & $9302(3)$ & $49(1)$ \\
\hline$C(45)$ & 1337(3) & $4303(2)$ & $7014(2)$ & $36(1)$ \\
\hline$C(46)$ & 1074(3) & $4848(2)$ & $6272(2)$ & $44(1)$ \\
\hline$C(47)$ & $495(3)$ & $4308(3)$ & $7268(3)$ & $61(1)$ \\
\hline$C(48)$ & $1406(3)$ & $3564(2)$ & $6674(3)$ & $46(1)$ \\
\hline C(49) & $3003(3)$ & $6284(2)$ & $8622(2)$ & $32(1)$ \\
\hline$C(50)$ & $2802(3)$ & $6496(2)$ & $9299(2)$ & $43(1)$ \\
\hline$C(51)$ & $3451(4)$ & $6919(2)$ & $9945(3)$ & $49(1)$ \\
\hline$C(52)$ & $4304(4)$ & $7124(2)$ & $9935(3)$ & $52(1)$ \\
\hline$C(53)$ & $4507(3)$ & $6919(2)$ & $9262(3)$ & $45(1)$ \\
\hline$C(54)$ & $3852(3)$ & $6498(2)$ & $8598(2)$ & $38(1)$ \\
\hline
\end{tabular}


Table 13. Bond lengths $[\AA]$ and angles $\left[{ }^{\circ}\right]$ for $\mathbf{2 d}$.

\begin{tabular}{|c|c|c|c|}
\hline $\operatorname{Pd}(1)-C(49)$ & $1.969(3)$ & $\mathrm{C}(11)-\mathrm{C}(16)$ & $1.390(5)$ \\
\hline $\operatorname{Pd}(1)-H(46 C)$ & 2.1282 & $\mathrm{C}(11)-\mathrm{C}(12)$ & $1.392(5)$ \\
\hline $\operatorname{Pd}(1)-\mathrm{P}(1)$ & $2.2567(10)$ & $\mathrm{C}(12)-\mathrm{C}(13)$ & $1.390(5)$ \\
\hline $\operatorname{Pd}(1)-\operatorname{Br}(1)$ & $2.4292(6)$ & $\mathrm{C}(13)-\mathrm{C}(14)$ & $1.362(5)$ \\
\hline $\mathrm{Fe}(1)-\operatorname{Cent}(1)$ & $1.683(4)$ & $\mathrm{C}(14)-\mathrm{C}(15)$ & $1.385(6)$ \\
\hline $\mathrm{Fe}(1)-\operatorname{Cent}(2)$ & $1.677(4)$ & $\mathrm{C}(15)-\mathrm{C}(16)$ & $1.381(5)$ \\
\hline $\mathrm{Fe}(1)-\mathrm{C}(9)$ & $2.049(4)$ & $\mathrm{C}(17)-\mathrm{C}(22)$ & $1.379(5)$ \\
\hline $\mathrm{Fe}(1)-\mathrm{C}(4)$ & $2.049(3)$ & $\mathrm{C}(17)-\mathrm{C}(18)$ & $1.390(5)$ \\
\hline $\mathrm{Fe}(1)-\mathrm{C}(10)$ & $2.053(3)$ & $\mathrm{C}(18)-\mathrm{C}(19)$ & $1.399(5)$ \\
\hline $\mathrm{Fe}(1)-\mathrm{C}(3)$ & $2.054(3)$ & $\mathrm{C}(19)-\mathrm{C}(20)$ & $1.367(6)$ \\
\hline $\mathrm{Fe}(1)-\mathrm{C}(8)$ & $2.060(3)$ & $\mathrm{C}(20)-\mathrm{C}(21)$ & $1.386(6)$ \\
\hline $\mathrm{Fe}(1)-\mathrm{C}(5)$ & $2.056(3)$ & $\mathrm{C}(21)-\mathrm{C}(22)$ & $1.377(5)$ \\
\hline $\mathrm{Fe}(1)-\mathrm{C}(6)$ & $2.077(3)$ & $\mathrm{C}(23)-\mathrm{C}(24)$ & $1.379(5)$ \\
\hline $\mathrm{Fe}(1)-\mathrm{C}(2)$ & $2.084(3)$ & $\mathrm{C}(23)-\mathrm{C}(28)$ & $1.396(5)$ \\
\hline $\mathrm{Fe}(1)-\mathrm{C}(7)$ & $2.098(3)$ & $\mathrm{C}(24)-\mathrm{C}(25)$ & $1.391(5)$ \\
\hline $\mathrm{Fe}(1)-\mathrm{C}(1)$ & $2.125(3)$ & $\mathrm{C}(25)-\mathrm{C}(26)$ & $1.374(6)$ \\
\hline $\mathrm{P}(1)-\mathrm{C}(1)$ & $1.807(4)$ & $C(26)-C(27)$ & $1.376(6)$ \\
\hline$P(1)-C(45)$ & $1.877(4)$ & $\mathrm{C}(27)-\mathrm{C}(28)$ & $1.385(5)$ \\
\hline$P(1)-C(41)$ & $1.891(4)$ & $\mathrm{C}(29)-\mathrm{C}(30)$ & $1.383(5)$ \\
\hline $\mathrm{C}(1)-\mathrm{C}(2)$ & $1.435(5)$ & $\mathrm{C}(29)-\mathrm{C}(34)$ & $1.401(5)$ \\
\hline$C(1)-C(5)$ & $1.436(5)$ & $\mathrm{C}(30)-\mathrm{C}(31)$ & $1.382(5)$ \\
\hline$C(2)-C(3)$ & $1.415(5)$ & $\mathrm{C}(31)-\mathrm{C}(32)$ & $1.373(6)$ \\
\hline$C(3)-C(4)$ & $1.427(5)$ & $\mathrm{C}(32)-\mathrm{C}(33)$ & $1.399(6)$ \\
\hline$C(4)-C(5)$ & $1.413(5)$ & $\mathrm{C}(33)-\mathrm{C}(34)$ & $1.381(5)$ \\
\hline$C(6)-C(10)$ & $1.442(5)$ & $C(35)-C(40)$ & $1.388(5)$ \\
\hline$C(6)-C(7)$ & $1.441(5)$ & $\mathrm{C}(35)-\mathrm{C}(36)$ & $1.399(5)$ \\
\hline$C(6)-C(11)$ & $1.481(5)$ & $\mathrm{C}(36)-\mathrm{C}(37)$ & $1.376(5)$ \\
\hline$C(7)-C(8)$ & $1.441(5)$ & $\mathrm{C}(37)-\mathrm{C}(38)$ & $1.391(6)$ \\
\hline$C(7)-C(17)$ & $1.488(5)$ & $\mathrm{C}(38)-\mathrm{C}(39)$ & $1.370(6)$ \\
\hline $\mathrm{C}(8)-\mathrm{C}(9)$ & $1.431(5)$ & $\mathrm{C}(39)-\mathrm{C}(40)$ & $1.385(5)$ \\
\hline $\mathrm{C}(8)-\mathrm{C}(23)$ & $1.490(5)$ & $\mathrm{C}(41)-\mathrm{C}(42)$ & $1.537(6)$ \\
\hline $\mathrm{C}(9)-\mathrm{C}(10)$ & $1.440(5)$ & $\mathrm{C}(41)-\mathrm{C}(43)$ & $1.542(5)$ \\
\hline$C(9)-C(29)$ & $1.499(5)$ & $\mathrm{C}(41)-\mathrm{C}(44)$ & $1.545(5)$ \\
\hline $\mathrm{C}(10)-\mathrm{C}(35)$ & $1.494(5)$ & $\mathrm{C}(45)-\mathrm{C}(48)$ & $1.540(5)$ \\
\hline
\end{tabular}




\begin{tabular}{|c|c|c|c|}
\hline$C(45)-C(47)$ & $1.539(6)$ & $\mathrm{C}(5)-\mathrm{Fe}(1)-\mathrm{C}(6)$ & $123.79(14)$ \\
\hline$C(45)-C(46)$ & $1.551(5)$ & $\mathrm{C}(9)-\mathrm{Fe}(1)-\mathrm{C}(2)$ & $144.81(14)$ \\
\hline$C(49)-C(50)$ & $1.392(5)$ & $\mathrm{C}(4)-\mathrm{Fe}(1)-\mathrm{C}(2)$ & $67.56(14)$ \\
\hline $\mathrm{C}(49)-\mathrm{C}(54)$ & $1.385(5)$ & $\mathrm{C}(10)-\mathrm{Fe}(1)-\mathrm{C}(2)$ & $173.56(14)$ \\
\hline$C(50)-C(51)$ & $1.377(6)$ & $\mathrm{C}(3)-\mathrm{Fe}(1)-\mathrm{C}(2)$ & $39.99(14)$ \\
\hline $\mathrm{C}(51)-\mathrm{C}(52)$ & $1.375(6)$ & $\mathrm{C}(8)-\mathrm{Fe}(1)-\mathrm{C}(2)$ & $117.18(14)$ \\
\hline$C(52)-C(53)$ & $1.383(6)$ & $\mathrm{C}(5)-\mathrm{Fe}(1)-\mathrm{C}(2)$ & $67.44(14)$ \\
\hline \multirow[t]{2}{*}{$C(53)-C(54)$} & $1.395(5)$ & $\mathrm{C}(6)-\mathrm{Fe}(1)-\mathrm{C}(2)$ & $137.97(14)$ \\
\hline & & $\mathrm{C}(9)-\mathrm{Fe}(1)-\mathrm{C}(7)$ & $68.20(14)$ \\
\hline $\mathrm{C}(49)-\mathrm{Pd}(1)-\mathrm{H}(46 \mathrm{C})$ & 176.6 & $\mathrm{C}(4)-\mathrm{Fe}(1)-\mathrm{C}(7)$ & $159.90(14)$ \\
\hline $\mathrm{C}(49)-\mathrm{Pd}(1)-\mathrm{P}(1)$ & $100.71(11)$ & $\mathrm{C}(10)-\mathrm{Fe}(1)-\mathrm{C}(7)$ & $68.48(13)$ \\
\hline $\mathrm{H}(46 \mathrm{C})-\mathrm{Pd}(1)-\mathrm{P}(1)$ & 79.9 & $\mathrm{C}(3)-\mathrm{Fe}(1)-\mathrm{C}(7)$ & $127.61(14)$ \\
\hline $\mathrm{C}(49)-\operatorname{Pd}(1)-\mathrm{Br}(1)$ & $91.94(11)$ & $\mathrm{C}(8)-\mathrm{Fe}(1)-\mathrm{C}(7)$ & $40.54(13)$ \\
\hline $\mathrm{H}(46 \mathrm{C})-\mathrm{Pd}(1)-\mathrm{Br}(1)$ & 87.8 & $\mathrm{C}(5)-\mathrm{Fe}(1)-\mathrm{C}(7)$ & $159.81(14)$ \\
\hline $\mathrm{P}(1)-\operatorname{Pd}(1)-\mathrm{Br}(1)$ & $166.16(3)$ & $\mathrm{C}(6)-\mathrm{Fe}(1)-\mathrm{C}(7)$ & $40.38(13)$ \\
\hline Cent(1)-Fe(1)-Cent(2) & $169.8(3)$ & $\mathrm{C}(2)-\mathrm{Fe}(1)-\mathrm{C}(7)$ & $114.67(14)$ \\
\hline $\mathrm{C}(9)-\mathrm{Fe}(1)-\mathrm{C}(4)$ & $98.22(14)$ & $\mathrm{C}(9)-\mathrm{Fe}(1)-\mathrm{C}(1)$ & $162.84(13)$ \\
\hline $\mathrm{C}(9)-\mathrm{Fe}(1)-\mathrm{C}(10)$ & $41.12(14)$ & $\mathrm{C}(4)-\mathrm{Fe}(1)-\mathrm{C}(1)$ & $67.43(14)$ \\
\hline $\mathrm{C}(4)-\mathrm{Fe}(1)-\mathrm{C}(10)$ & $111.59(14)$ & $\mathrm{C}(10)-\mathrm{Fe}(1)-\mathrm{C}(1)$ & $133.70(13)$ \\
\hline $\mathrm{C}(9)-\mathrm{Fe}(1)-\mathrm{C}(3)$ & $108.43(15)$ & $\mathrm{C}(3)-\mathrm{Fe}(1)-\mathrm{C}(1)$ & $67.36(14)$ \\
\hline $\mathrm{C}(4)-\mathrm{Fe}(1)-\mathrm{C}(3)$ & $40.70(13)$ & $\mathrm{C}(8)-\mathrm{Fe}(1)-\mathrm{C}(1)$ & $154.62(13)$ \\
\hline $\mathrm{C}(10)-\mathrm{Fe}(1)-\mathrm{C}(3)$ & $143.10(14)$ & $\mathrm{C}(5)-\mathrm{Fe}(1)-\mathrm{C}(1)$ & $40.13(13)$ \\
\hline $\mathrm{C}(9)-\mathrm{Fe}(1)-\mathrm{C}(8)$ & $40.76(14)$ & $\mathrm{C}(6)-\mathrm{Fe}(1)-\mathrm{C}(1)$ & $119.03(13)$ \\
\hline $\mathrm{C}(4)-\mathrm{Fe}(1)-\mathrm{C}(8)$ & 119.67(14) & $\mathrm{C}(2)-\mathrm{Fe}(1)-\mathrm{C}(1)$ & $39.86(13)$ \\
\hline $\mathrm{C}(10)-\mathrm{Fe}(1)-\mathrm{C}(8)$ & $69.01(14)$ & $\mathrm{C}(7)-\mathrm{Fe}(1)-\mathrm{C}(1)$ & $128.11(13)$ \\
\hline $\mathrm{C}(3)-\mathrm{Fe}(1)-\mathrm{C}(8)$ & $101.42(14)$ & $\mathrm{C}(1)-\mathrm{P}(1)-\mathrm{C}(45)$ & $110.97(17)$ \\
\hline $\mathrm{C}(9)-\mathrm{Fe}(1)-\mathrm{C}(5)$ & $122.72(14)$ & $\mathrm{C}(1)-\mathrm{P}(1)-\mathrm{C}(41)$ & $101.68(17)$ \\
\hline $\mathrm{C}(4)-\mathrm{Fe}(1)-\mathrm{C}(5)$ & $40.27(13)$ & $\mathrm{C}(45)-\mathrm{P}(1)-\mathrm{C}(41)$ & $111.58(18)$ \\
\hline $\mathrm{C}(10)-\mathrm{Fe}(1)-\mathrm{C}(5)$ & $107.58(14)$ & $\mathrm{C}(1)-\mathrm{P}(1)-\mathrm{Pd}(1)$ & $118.68(11)$ \\
\hline $\mathrm{C}(3)-\mathrm{Fe}(1)-\mathrm{C}(5)$ & $67.95(14)$ & $\mathrm{C}(45)-\mathrm{P}(1)-\mathrm{Pd}(1)$ & $94.52(12)$ \\
\hline $\mathrm{C}(8)-\mathrm{Fe}(1)-\mathrm{C}(5)$ & $158.41(14)$ & $\mathrm{C}(41)-\mathrm{P}(1)-\mathrm{Pd}(1)$ & $119.57(12)$ \\
\hline $\mathrm{C}(9)-\mathrm{Fe}(1)-\mathrm{C}(6)$ & $68.55(14)$ & $\mathrm{C}(2)-\mathrm{C}(1)-\mathrm{C}(5)$ & $106.3(3)$ \\
\hline $\mathrm{C}(4)-\mathrm{Fe}(1)-\mathrm{C}(6)$ & $149.78(14)$ & $\mathrm{C}(2)-\mathrm{C}(1)-\mathrm{P}(1)$ & $128.4(3)$ \\
\hline $\mathrm{C}(10)-\mathrm{Fe}(1)-\mathrm{C}(6)$ & $40.86(13)$ & $\mathrm{C}(5)-\mathrm{C}(1)-\mathrm{P}(1)$ & $119.8(2)$ \\
\hline $\mathrm{C}(3)-\mathrm{Fe}(1)-\mathrm{C}(6)$ & $167.96(14)$ & $\mathrm{C}(2)-\mathrm{C}(1)-\mathrm{Fe}(1)$ & $68.53(19)$ \\
\hline $\mathrm{C}(8)-\mathrm{Fe}(1)-\mathrm{C}(6)$ & $68.43(13)$ & $\mathrm{C}(5)-\mathrm{C}(1)-\mathrm{Fe}(1)$ & $67.34(19)$ \\
\hline
\end{tabular}




\begin{tabular}{|c|c|c|c|}
\hline $\mathrm{P}(1)-\mathrm{C}(1)-\mathrm{Fe}(1)$ & $149.29(18)$ & $\mathrm{C}(29)-\mathrm{C}(9)-\mathrm{Fe}(1)$ & $129.1(2)$ \\
\hline$C(3)-C(2)-C(1)$ & $108.8(3)$ & $C(9)-C(10)-C(6)$ & $107.5(3)$ \\
\hline $\mathrm{C}(3)-\mathrm{C}(2)-\mathrm{Fe}(1)$ & $68.9(2)$ & $C(9)-C(10)-C(35)$ & $127.4(3)$ \\
\hline $\mathrm{C}(1)-\mathrm{C}(2)-\mathrm{Fe}(1)$ & 71.61(19) & $C(6)-C(10)-C(35)$ & $124.9(3)$ \\
\hline $\mathrm{C}(2)-\mathrm{C}(3)-\mathrm{C}(4)$ & $107.9(3)$ & $\mathrm{C}(9)-\mathrm{C}(10)-\mathrm{Fe}(1)$ & 69.31(19) \\
\hline $\mathrm{C}(2)-\mathrm{C}(3)-\mathrm{Fe}(1)$ & $71.1(2)$ & $\mathrm{C}(6)-\mathrm{C}(10)-\mathrm{Fe}(1)$ & $70.46(19)$ \\
\hline $\mathrm{C}(4)-\mathrm{C}(3)-\mathrm{Fe}(1)$ & $69.46(19)$ & $\mathrm{C}(35)-\mathrm{C}(10)-\mathrm{Fe}(1)$ & $129.4(2)$ \\
\hline $\mathrm{C}(5)-\mathrm{C}(4)-\mathrm{C}(3)$ & $107.9(3)$ & $C(16)-C(11)-C(12)$ & $117.7(3)$ \\
\hline $\mathrm{C}(5)-\mathrm{C}(4)-\mathrm{Fe}(1)$ & $70.12(18)$ & $C(16)-C(11)-C(6)$ & $118.8(3)$ \\
\hline $\mathrm{C}(3)-\mathrm{C}(4)-\mathrm{Fe}(1)$ & $69.84(19)$ & $C(12)-C(11)-C(6)$ & $123.4(3)$ \\
\hline $\mathrm{C}(4)-\mathrm{C}(5)-\mathrm{C}(1)$ & $108.9(3)$ & $C(13)-C(12)-C(11)$ & $121.2(3)$ \\
\hline $\mathrm{C}(4)-\mathrm{C}(5)-\mathrm{Fe}(1)$ & 69.61(19) & $C(14)-C(13)-C(12)$ & $119.8(4)$ \\
\hline $\mathrm{C}(1)-\mathrm{C}(5)-\mathrm{Fe}(1)$ & $72.53(19)$ & $\mathrm{C}(13)-\mathrm{C}(14)-\mathrm{C}(15)$ & $120.2(4)$ \\
\hline$C(10)-C(6)-C(7)$ & $108.3(3)$ & $C(16)-C(15)-C(14)$ & $120.0(4)$ \\
\hline$C(10)-C(6)-C(11)$ & $125.2(3)$ & $C(15)-C(16)-C(11)$ & $121.0(4)$ \\
\hline $\mathrm{C}(7)-\mathrm{C}(6)-\mathrm{C}(11)$ & $125.6(3)$ & $\mathrm{C}(22)-\mathrm{C}(17)-\mathrm{C}(18)$ & $118.3(3)$ \\
\hline $\mathrm{C}(10)-\mathrm{C}(6)-\mathrm{Fe}(1)$ & $68.68(18)$ & $\mathrm{C}(22)-\mathrm{C}(17)-\mathrm{C}(7)$ & $121.9(3)$ \\
\hline $\mathrm{C}(7)-\mathrm{C}(6)-\mathrm{Fe}(1)$ & $70.59(18)$ & $\mathrm{C}(18)-\mathrm{C}(17)-\mathrm{C}(7)$ & $119.5(3)$ \\
\hline $\mathrm{C}(11)-\mathrm{C}(6)-\mathrm{Fe}(1)$ & $134.7(2)$ & $\mathrm{C}(17)-\mathrm{C}(18)-\mathrm{C}(19)$ & $120.4(4)$ \\
\hline $\mathrm{C}(8)-\mathrm{C}(7)-\mathrm{C}(6)$ & $107.7(3)$ & $\mathrm{C}(20)-\mathrm{C}(19)-\mathrm{C}(18)$ & $120.3(4)$ \\
\hline $\mathrm{C}(8)-\mathrm{C}(7)-\mathrm{C}(17)$ & $126.3(3)$ & $C(19)-C(20)-C(21)$ & $119.5(4)$ \\
\hline$C(6)-C(7)-C(17)$ & $125.2(3)$ & $C(22)-C(21)-C(20)$ & $120.2(4)$ \\
\hline $\mathrm{C}(8)-\mathrm{C}(7)-\mathrm{Fe}(1)$ & $68.32(18)$ & $C(17)-C(22)-C(21)$ & $121.3(4)$ \\
\hline$C(6)-C(7)-F e(1)$ & $69.04(17)$ & $C(24)-C(23)-C(28)$ & $118.5(3)$ \\
\hline $\mathrm{C}(17)-\mathrm{C}(7)-\mathrm{Fe}(1)$ & $136.0(2)$ & $\mathrm{C}(24)-\mathrm{C}(23)-\mathrm{C}(8)$ & $119.3(3)$ \\
\hline C(9)-C(8)-C(7) & 108.1(3) & $\mathrm{C}(28)-\mathrm{C}(23)-\mathrm{C}(8)$ & $122.1(3)$ \\
\hline $\mathrm{C}(9)-\mathrm{C}(8)-\mathrm{C}(23)$ & $127.2(3)$ & $\mathrm{C}(23)-\mathrm{C}(24)-\mathrm{C}(25)$ & $121.2(4)$ \\
\hline $\mathrm{C}(7)-\mathrm{C}(8)-\mathrm{C}(23)$ & $124.4(3)$ & $C(26)-C(25)-C(24)$ & $119.5(4)$ \\
\hline $\mathrm{C}(9)-\mathrm{C}(8)-\mathrm{Fe}(1)$ & $69.22(19)$ & $C(27)-C(26)-C(25)$ & $120.4(4)$ \\
\hline $\mathrm{C}(7)-\mathrm{C}(8)-\mathrm{Fe}(1)$ & 71.14(19) & $C(26)-C(27)-C(28)$ & $120.1(4)$ \\
\hline $\mathrm{C}(23)-\mathrm{C}(8)-\mathrm{Fe}(1)$ & $130.0(2)$ & $\mathrm{C}(27)-\mathrm{C}(28)-\mathrm{C}(23)$ & $120.4(4)$ \\
\hline $\mathrm{C}(8)-\mathrm{C}(9)-\mathrm{C}(10)$ & $108.4(3)$ & $C(30)-C(29)-C(34)$ & 118.2(3) \\
\hline $\mathrm{C}(8)-\mathrm{C}(9)-\mathrm{C}(29)$ & $125.8(3)$ & $\mathrm{C}(30)-\mathrm{C}(29)-\mathrm{C}(9)$ & $120.1(3)$ \\
\hline$C(10)-C(9)-C(29)$ & $125.6(3)$ & $\mathrm{C}(34)-\mathrm{C}(29)-\mathrm{C}(9)$ & $121.6(3)$ \\
\hline $\mathrm{C}(8)-\mathrm{C}(9)-\mathrm{Fe}(1)$ & $70.0(2)$ & $\mathrm{C}(31)-\mathrm{C}(30)-\mathrm{C}(29)$ & $121.5(4)$ \\
\hline $\mathrm{C}(10)-\mathrm{C}(9)-\mathrm{Fe}(1)$ & 69.57(19) & $C(32)-C(31)-C(30)$ & $120.4(4)$ \\
\hline
\end{tabular}




$\begin{array}{ll}\mathrm{C}(31)-\mathrm{C}(32)-\mathrm{C}(33) & 119.2(4) \\ \mathrm{C}(34)-\mathrm{C}(33)-\mathrm{C}(32) & 120.4(4) \\ \mathrm{C}(33)-\mathrm{C}(34)-\mathrm{C}(29) & 120.4(4) \\ \mathrm{C}(40)-\mathrm{C}(35)-\mathrm{C}(36) & 118.1(3) \\ \mathrm{C}(40)-\mathrm{C}(35)-\mathrm{C}(10) & 120.4(3) \\ \mathrm{C}(36)-\mathrm{C}(35)-\mathrm{C}(10) & 121.4(3) \\ \mathrm{C}(37)-\mathrm{C}(36)-\mathrm{C}(35) & 120.6(4) \\ \mathrm{C}(36)-\mathrm{C}(37)-\mathrm{C}(38) & 120.7(4) \\ \mathrm{C}(39)-\mathrm{C}(38)-\mathrm{C}(37) & 119.0(4) \\ \mathrm{C}(38)-\mathrm{C}(39)-\mathrm{C}(40) & 120.8(4) \\ \mathrm{C}(39)-\mathrm{C}(40)-\mathrm{C}(35) & 120.9(3) \\ \mathrm{C}(42)-\mathrm{C}(41)-\mathrm{C}(43) & 108.7(3) \\ \mathrm{C}(42)-\mathrm{C}(41)-\mathrm{C}(44) & 107.6(3) \\ \mathrm{C}(43)-\mathrm{C}(41)-\mathrm{C}(44) & 110.0(3) \\ \mathrm{C}(42)-\mathrm{C}(41)-\mathrm{P}(1) & 107.8(3) \\ \mathrm{C}(43)-\mathrm{C}(41)-\mathrm{P}(1) & 112.0(3) \\ \mathrm{C}(44)-\mathrm{C}(41)-\mathrm{P}(1) & 110.5(3) \\ \mathrm{C}(48)-\mathrm{C}(45)-\mathrm{C}(47) & 108.7(3) \\ \mathrm{C}(48)-\mathrm{C}(45)-\mathrm{C}(46) & 109.2(3) \\ \mathrm{C}(47)-\mathrm{C}(45)-\mathrm{C}(46) & 106.5(3) \\ \mathrm{C}(48)-\mathrm{C}(45)-\mathrm{P}(1) & 117.2(3) \\ \mathrm{C}(47)-\mathrm{C}(45)-\mathrm{P}(1) & 110.6(3) \\ \mathrm{C}(46)-\mathrm{C}(45)-\mathrm{P}(1) & 104.0(2) \\ \mathrm{C}(50)-\mathrm{C}(49)-\mathrm{C}(54) & 120.0(3) \\ \mathrm{C}(50)-\mathrm{C}(49)-\mathrm{Pd}(1) & 120.0(3) \\ \mathrm{C}(54)-\mathrm{C}(49)-\mathrm{Pd}(1) & 119.3(3) \\ \mathrm{C}(51)-\mathrm{C}(50)-\mathrm{C}(49) & 119.7(4) \\ \mathrm{C}(52)-\mathrm{C}(51)-\mathrm{C}(50) & 120.7(4) \\ \mathrm{C}(51)-\mathrm{C}(52)-\mathrm{C}(53) & 120.0(4) \\ \mathrm{C}(52)-\mathrm{C}(53)-\mathrm{C}(54) & 119.9(4) \\ \mathrm{C}(49)-\mathrm{C}(54)-\mathrm{C}(53) & 119.6(4)\end{array}$


Table 14. Anisotropic displacement parameters $\left(\AA^{2} \times 10^{3}\right)$ for $\mathbf{2 d}$. The anisotropic displacement factor exponent takes the form: $-2 \pi^{2}\left[h^{2} a^{* 2} U^{11}+\ldots+2 h k a^{*} b^{*} U^{12}\right]$

\begin{tabular}{|c|c|c|c|c|c|c|}
\hline & $\mathrm{U}^{11}$ & $\mathrm{U}^{22}$ & $\mathrm{U}^{33}$ & $\mathrm{U}^{23}$ & $\mathrm{U}^{13}$ & $\mathrm{U}^{12}$ \\
\hline $\operatorname{Pd}(1)$ & $29(1)$ & $28(1)$ & $33(1)$ & $-3(1)$ & $10(1)$ & $3(1)$ \\
\hline $\operatorname{Br}(1)$ & $40(1)$ & $32(1)$ & $45(1)$ & $-2(1)$ & $11(1)$ & $9(1)$ \\
\hline $\mathrm{Fe}(1)$ & $26(1)$ & $23(1)$ & $24(1)$ & $1(1)$ & $10(1)$ & $-1(1)$ \\
\hline $\mathrm{P}(1)$ & $30(1)$ & $26(1)$ & $30(1)$ & $-3(1)$ & $13(1)$ & $0(1)$ \\
\hline $\mathrm{C}(1)$ & $27(2)$ & $25(2)$ & $24(2)$ & $4(1)$ & $12(2)$ & $1(2)$ \\
\hline $\mathrm{C}(2)$ & $37(2)$ & $22(2)$ & $32(2)$ & $3(2)$ & $19(2)$ & $2(2)$ \\
\hline$C(3)$ & $34(2)$ & $28(2)$ & $30(2)$ & $5(2)$ & $15(2)$ & $4(2)$ \\
\hline $\mathrm{C}(4)$ & $32(2)$ & $31(2)$ & $22(2)$ & $5(2)$ & $8(2)$ & $3(2)$ \\
\hline$C(5)$ & $35(2)$ & $29(2)$ & $26(2)$ & $-1(2)$ & $16(2)$ & $0(2)$ \\
\hline$C(6)$ & $29(2)$ & $26(2)$ & $24(2)$ & $2(2)$ & $14(2)$ & $-3(2)$ \\
\hline$C(7)$ & $27(2)$ & $26(2)$ & $19(2)$ & $0(1)$ & $9(2)$ & $-3(2)$ \\
\hline $\mathrm{C}(8)$ & $31(2)$ & $29(2)$ & $24(2)$ & $1(2)$ & $13(2)$ & $-3(2)$ \\
\hline $\mathrm{C}(9)$ & $27(2)$ & $31(2)$ & $29(2)$ & $3(2)$ & $15(2)$ & $-2(2)$ \\
\hline$C(10)$ & $31(2)$ & $26(2)$ & $20(2)$ & $1(1)$ & $11(2)$ & $-5(2)$ \\
\hline $\mathrm{C}(11)$ & $32(2)$ & $24(2)$ & $28(2)$ & $0(2)$ & $14(2)$ & $-5(2)$ \\
\hline$C(12)$ & $47(2)$ & $29(2)$ & $31(2)$ & $2(2)$ & $20(2)$ & $0(2)$ \\
\hline$C(13)$ & $49(3)$ & $31(2)$ & $55(3)$ & $3(2)$ & $29(2)$ & $7(2)$ \\
\hline$C(14)$ & $33(2)$ & $38(2)$ & $53(3)$ & $13(2)$ & $18(2)$ & $9(2)$ \\
\hline$C(15)$ & $31(2)$ & $43(2)$ & $37(2)$ & $12(2)$ & $12(2)$ & $1(2)$ \\
\hline$C(16)$ & $31(2)$ & $29(2)$ & $32(2)$ & $4(2)$ & $14(2)$ & $3(2)$ \\
\hline$C(17)$ & $34(2)$ & $22(2)$ & $26(2)$ & $3(2)$ & $12(2)$ & $-5(2)$ \\
\hline $\mathrm{C}(18)$ & $31(2)$ & $35(2)$ & $32(2)$ & $-2(2)$ & $13(2)$ & $1(2)$ \\
\hline $\mathrm{C}(19)$ & $61(3)$ & $41(2)$ & $32(2)$ & $-10(2)$ & $22(2)$ & $-8(2)$ \\
\hline $\mathrm{C}(20)$ & $44(3)$ & $39(2)$ & $35(2)$ & $-2(2)$ & $5(2)$ & $-13(2)$ \\
\hline $\mathrm{C}(21)$ & $32(2)$ & $40(2)$ & $46(2)$ & $-1(2)$ & $10(2)$ & $-4(2)$ \\
\hline $\mathrm{C}(22)$ & $33(2)$ & $37(2)$ & $35(2)$ & $-6(2)$ & $15(2)$ & $-4(2)$ \\
\hline $\mathrm{C}(23)$ & $27(2)$ & $28(2)$ & $24(2)$ & $1(2)$ & $9(2)$ & $4(2)$ \\
\hline $\mathrm{C}(24)$ & $33(2)$ & $35(2)$ & $44(2)$ & $-2(2)$ & $18(2)$ & $-2(2)$ \\
\hline $\mathrm{C}(25)$ & $46(3)$ & $48(3)$ & $63(3)$ & $1(2)$ & $35(2)$ & $12(2)$ \\
\hline
\end{tabular}




$\begin{array}{lllllll}\mathrm{C}(26) & 64(3) & 37(3) & 60(3) & 2(2) & 29(3) & 19(2) \\ \mathrm{C}(27) & 54(3) & 30(2) & 56(3) & 2(2) & 26(2) & -5(2) \\ \mathrm{C}(28) & 34(2) & 32(2) & 42(2) & 2(2) & 22(2) & 2(2) \\ \mathrm{C}(29) & 27(2) & 36(2) & 28(2) & -6(2) & 11(2) & -5(2) \\ \mathrm{C}(30) & 32(2) & 40(2) & 36(2) & -3(2) & 16(2) & -4(2) \\ \mathrm{C}(31) & 29(2) & 66(3) & 53(3) & -11(2) & 19(2) & -13(2) \\ \mathrm{C}(32) & 24(2) & 81(4) & 54(3) & -8(3) & 7(2) & -1(2) \\ \mathrm{C}(33) & 40(3) & 57(3) & 47(3) & 7(2) & 11(2) & 8(2) \\ \mathrm{C}(34) & 25(2) & 44(2) & 39(2) & 1(2) & 11(2) & -3(2) \\ \mathrm{C}(35) & 33(2) & 24(2) & 34(2) & -3(2) & 18(2) & -5(2) \\ \mathrm{C}(36) & 39(2) & 36(2) & 33(2) & -3(2) & 16(2) & -9(2) \\ \mathrm{C}(37) & 51(3) & 48(3) & 36(2) & -12(2) & 22(2) & -17(2) \\ \mathrm{C}(38) & 61(3) & 34(2) & 58(3) & -17(2) & 32(2) & -19(2) \\ \mathrm{C}(39) & 54(3) & 34(2) & 51(3) & 1(2) & 25(2) & -9(2) \\ \mathrm{C}(40) & 36(2) & 31(2) & 36(2) & 0(2) & 16(2) & -5(2) \\ \mathrm{C}(41) & 43(2) & 36(2) & 33(2) & 4(2) & 20(2) & 1(2) \\ \mathrm{C}(42) & 55(3) & 47(2) & 28(2) & 6(2) & 19(2) & 2(2) \\ \mathrm{C}(43) & 64(3) & 38(2) & 51(3) & 10(2) & 33(2) & 1(2) \\ \mathrm{C}(44) & 52(3) & 56(3) & 50(3) & -5(2) & 34(2) & -2(2) \\ \mathrm{C}(45) & 25(2) & 39(2) & 40(2) & -6(2) & 10(2) & -6(2) \\ \mathrm{C}(46) & 41(2) & 39(2) & 39(2) & -5(2) & 6(2) & 4(2) \\ \mathrm{C}(47) & 34(3) & 89(4) & 60(3) & -20(3) & 21(2) & -12(2) \\ \mathrm{C}(48) & 50(3) & 38(2) & 42(2) & -6(2) & 14(2) & -10(2) \\ \mathrm{C}(49) & 31(2) & 27(2) & 31(2) & -3(2) & 9(2) & 0(2) \\ \mathrm{C}(50) & 47(3) & 38(2) & 41(2) & -5(2) & 18(2) & 0(2) \\ \mathrm{C}(51) & 66(3) & 39(2) & 39(2) & -3(2) & 21(2) & 4(2) \\ \mathrm{C}(52) & 64(3) & 29(2) & 36(2) & -5(2) & -2(2) & 6(2) \\ \mathrm{C}(53) & 36(2) & 30(2) & 51(3) & 3(2) & 2(2) & 2(2) \\ \mathrm{C}(54) & 36(2) & 29(2) & 43(2) & -4(2) & 13(2) & 2(2)\end{array}$


Table 15. Hydrogen coordinates $\left(x 10^{4}\right)$ and isotropic displacement parameters $\left(\AA^{2} \mathrm{x}\right.$ $10^{3}$ )for $2 \mathbf{d}$.

\begin{tabular}{|c|c|c|c|c|}
\hline- & $\mathrm{X}$ & $\mathrm{y}$ & $\mathrm{z}$ & $\mathrm{U}(\mathrm{eq})$ \\
\hline $\mathrm{H}(2 \mathrm{~A})$ & 3308 & 3294 & 7354 & 35 \\
\hline $\mathrm{H}(3 \mathrm{~A})$ & 5174 & 3176 & 8071 & 36 \\
\hline $\mathrm{H}(4 \mathrm{~A})$ & 5909 & 4387 & 8762 & 35 \\
\hline $\mathrm{H}(5 \mathrm{~A})$ & 4499 & 5254 & 8453 & 34 \\
\hline $\mathrm{H}(12 \mathrm{~A})$ & 3083 & 5954 & 6610 & 42 \\
\hline $\mathrm{H}(13 \mathrm{~A})$ & 1925 & 6809 & 5859 & 51 \\
\hline $\mathrm{H}(14 \mathrm{~A})$ & 1300 & 6885 & 4370 & 50 \\
\hline $\mathrm{H}(15 \mathrm{~A})$ & 1793 & 6085 & 3612 & 46 \\
\hline $\mathrm{H}(16 \mathrm{~A})$ & 2934 & 5222 & 4353 & 37 \\
\hline $\mathrm{H}(18 \mathrm{~A})$ & 3932 & 3463 & 4535 & 40 \\
\hline $\mathrm{H}(19 \mathrm{~A})$ & 2641 & 3005 & 3300 & 53 \\
\hline $\mathrm{H}(20 \mathrm{~A})$ & 1060 & 3153 & 3102 & 52 \\
\hline $\mathrm{H}(21 \mathrm{~A})$ & 761 & 3749 & 4150 & 50 \\
\hline $\mathrm{H}(22 \mathrm{~A})$ & 2035 & 4211 & 5364 & 42 \\
\hline $\mathrm{H}(24 \mathrm{~A})$ & 6291 & 3547 & 5935 & 44 \\
\hline $\mathrm{H}(25 \mathrm{~A})$ & 6737 & 2415 & 5707 & 58 \\
\hline $\mathrm{H}(26 \mathrm{~A})$ & 5919 & 1438 & 5872 & 64 \\
\hline $\mathrm{H}(27 \mathrm{~A})$ & 4639 & 1584 & 6228 & 55 \\
\hline $\mathrm{H}(28 \mathrm{~A})$ & 4168 & 2714 & 6425 & 41 \\
\hline $\mathrm{H}(30 \mathrm{~A})$ & 6895 & 5415 & 6916 & 43 \\
\hline $\mathrm{H}(31 \mathrm{~A})$ & 8562 & 5348 & 7717 & 59 \\
\hline $\mathrm{H}(32 \mathrm{~A})$ & 9260 & 4398 & 8639 & 68 \\
\hline $\mathrm{H}(33 \mathrm{~A})$ & 8262 & 3500 & 8743 & 61 \\
\hline $\mathrm{H}(34 \mathrm{~A})$ & 6591 & 3558 & 7925 & 45 \\
\hline $\mathrm{H}(36 \mathrm{~A})$ & 6027 & 5633 & 8242 & 43 \\
\hline $\mathrm{H}(37 \mathrm{~A})$ & 6584 & 6758 & 8742 & 53 \\
\hline $\mathrm{H}(38 \mathrm{~A})$ & 6151 & 7717 & 7784 & 59 \\
\hline $\mathrm{H}(39 \mathrm{~A})$ & 5128 & 7533 & 6331 & 55 \\
\hline $\mathrm{H}(40 \mathrm{~A})$ & 4585 & 6405 & 5814 & 41 \\
\hline $\mathrm{H}(42 \mathrm{~A})$ & 3836 & 4313 & 10226 & 65 \\
\hline
\end{tabular}




$\begin{array}{lrrrr}\mathrm{H}(42 B) & 3755 & 5020 & 9689 & 65 \\ \mathrm{H}(42 \mathrm{C}) & 4181 & 4315 & 9474 & 65 \\ \mathrm{H}(43 \mathrm{~A}) & 2727 & 3302 & 9545 & 73 \\ \mathrm{H}(43 \mathrm{~B}) & 3099 & 3281 & 8811 & 73 \\ \mathrm{H}(43 \mathrm{C}) & 1964 & 3334 & 8546 & 73 \\ \mathrm{H}(44 \mathrm{~A}) & 2080 & 4403 & 9865 & 73 \\ \mathrm{H}(44 \mathrm{~B}) & 1295 & 4494 & 8880 & 73 \\ \mathrm{H}(44 \mathrm{C}) & 2041 & 5118 & 9357 & 73 \\ \mathrm{H}(46 \mathrm{~A}) & 487 & 4694 & 5769 & 66 \\ \mathrm{H}(46 \mathrm{~B}) & 1610 & 4885 & 6111 & 66 \\ \mathrm{H}(46 \mathrm{C}) & 960 & 5310 & 6466 & 66 \\ \mathrm{H}(47 \mathrm{~A}) & -86 & 4111 & 6795 & 91 \\ \mathrm{H}(47 \mathrm{~B}) & 366 & 4794 & 7382 & 91 \\ \mathrm{H}(47 \mathrm{C}) & 670 & 4022 & 7790 & 91 \\ \mathrm{H}(48 \mathrm{~A}) & 775 & 3431 & 6211 & 69 \\ \mathrm{H}(48 \mathrm{~B}) & 1597 & 3221 & 7147 & 69 \\ \mathrm{H}(48 \mathrm{C}) & 1891 & 3569 & 6449 & 69 \\ \mathrm{H}(50 \mathrm{~A}) & 2220 & 6351 & 9316 & 51 \\ \mathrm{H}(51 \mathrm{~A}) & 3308 & 7071 & 10402 & 58 \\ \mathrm{H}(52 \mathrm{~A}) & 4753 & 7407 & 10391 & 63 \\ \mathrm{H}(53 \mathrm{~A}) & 5093 & 7065 & 9252 & 55 \\ \mathrm{H}(54 \mathrm{~A}) & 3987 & 6359 & 8132 & 45\end{array}$


